\title{
Probe Incompatibility in Multiparameter Noisy Quantum Metrology
}

\author{
Francesco Albarelli® ${ }^{1,2, *}$ and Rafał Demkowicz-Dobrzański® ${ }^{1}$ \\ ${ }^{1}$ Faculty of Physics, University of Warsaw, 02-093 Warszawa, Poland \\ ${ }^{2}$ Department of Physics, University of Warwick, Coventry CV4 7AL, United Kingdom
}

(Received 13 May 2021; revised 29 November 2021; accepted 6 January 2022; published 1 March 2022)

\begin{abstract}
We derive fundamental bounds on the maximal achievable precision in multiparameter noisy quantum metrology, valid under the most general entanglement-assisted adaptive strategy, which are tighter than the bounds obtained by a direct use of single-parameter results. This allows us to study the issue of the optimal probe incompatibility in the simultaneous estimation of multiple parameters in generic noisy channels, while so far the issue has been studied mostly in effectively noiseless scenarios (where the Heisenberg scaling is possible). We apply our results to the estimation of both unitary and noise parameters and indicate models where the fundamental probe incompatibility is present. In particular, we show that in lossy multiple-arm interferometry the probe incompatibility is as strong as in the noiseless scenario, reducing the potential advantage of simultaneous estimation to a constant factor. Finally, going beyond the multiparameter estimation paradigm, we introduce the concept of random quantum sensing and show how the tools developed may be applied to multiple-channel discrimination problems. As an illustration, we provide a simple proof of the loss of the quadratic advantage of the time-continuous Grover algorithm in the presence of dephasing or erasure noise.
\end{abstract}

DOI: $10.1103 /$ PhysRevX.12.011039

Subject Areas: Quantum Information

\section{INTRODUCTION}

Precise characterization of the parameters of physical systems is an important task, both from a technological as well as a purely scientific perspective. Understanding the limits on how precisely the parameters can be estimated, given the most general estimation protocols admitted by quantum mechanics, touches also upon the foundations of quantum mechanics itself. When it comes to single-parameter quantum metrology, both the theory and practical applications are now in their maturity stage. The theory provides not only the fundamental bounds that indicate in which models one may expect the most promising quantum enhancements [1-5], but it also provides explicit protocols based on the use of squeezed states and quantum-errorcorrection ideas to reach these limits [1,5-8]. The most spectacular application is the use of squeezed states of light in modern gravitational-wave detectors $[9,10]$, which operate surprisingly close to the fundamental limits [6], taking into account the level of optical losses present in the devices.

\footnotetext{
"Present address: Dipartimento di Fisica "Aldo Pontremoli", Università degli Studi di Milano, via Celoria 16, 20133 Milan, Italy.

Published by the American Physical Society under the terms of the Creative Commons Attribution 4.0 International license. Further distribution of this work must maintain attribution to the author(s) and the published article's title, journal citation, and DOI.
}

Multiparameter estimation problems are abundant, e.g., vector field estimation [11], multiarm interferometry [12], waveform estimation [13], etc., and this research domain has rightfully attracted an increasing amount of attention in recent years $[14,15]$. The aim of multiparameter quantum metrology is to obtain the most precise estimates of several parameters simultaneously, i.e., within a single experimental configuration. A quantum-metrology experiment can schematically be divided in three stages: the preparation of a probe state, the sensing stage when the probe's evolution is affected by the parameters of interest, and finally, the measurement. Assuming that the evolution of the probe is fixed by the physical nature of the problem, the final goal is to find a combination of probe state and measurement that achieves the best possible precision of estimating unknown parameters of the evolution. As such, a metrological problem may be regarded formally as a quantum-channel estimation problem. The multiparameter character, however, adds another layer of complexity on top of single-parameter scenarios, and the identification of fundamental bounds and optimal protocols becomes much more challenging.

In many practical situations, the channel can be probed many times, and the actual optimal protocols may be adaptive. Evaluating the power of adaptive strategies, especially in the presence of a noisy environment, is a challenging task appearing in various contexts throughout the whole field of quantum-information theory [16-18]. It is remarkable that this problem has been completely resolved in the case of single-parameter quantum metrology 
$[3-5,7,8]$, proving the effectiveness of the theoretical methods developed. The main goal of this paper is to generalize these methods to the multiparameter scenario.

The most intriguing aspect of multiparameter quantum metrology is the existence of protocols for simultaneous estimation that achieve a better overall precision than estimating each parameter separately, given the same amount of resources (e.g., number of particles, total sensing time). Indeed, several theoretical $[11,12,19-23]$ and experimental [24-27] studies have shown such an advantage in noiseless and error-corrected [28] scenarios. In the best case, it may be possible to estimate all parameters in a single experiment with the same precision obtainable in separate experiments for each parameter. The conditions for such a maximal advantage, known as compatibility conditions, are laid out in Ref. [29]. We report them here: "(i) existence of a single probe state allowing for optimal sensitivity for all parameters of interest, (ii) existence of a single measurement optimally extracting information from the probe state on all parameters, and (iii) statistical independence of the estimated parameters." These three aspects are pictorially represented in Fig. 1.

The impossibility to satisfy condition (ii) is known as measurement incompatibility, and it boils down to the fact that the optimal observables to estimate different parameters might not commute. This issue has always been central in quantum estimation theory [30-34]: from the seminal studies of almost half a century ago [35-37] to recent developments [38-46]. An important and relatively new observation is that measurement incompatibility will at most double the total mean-squared error on the parameters' estimates $[47,48]$ when a large number of identical copies of the probe state can be measured collectively [49-51]. Importantly, for the generic noisy protocols that

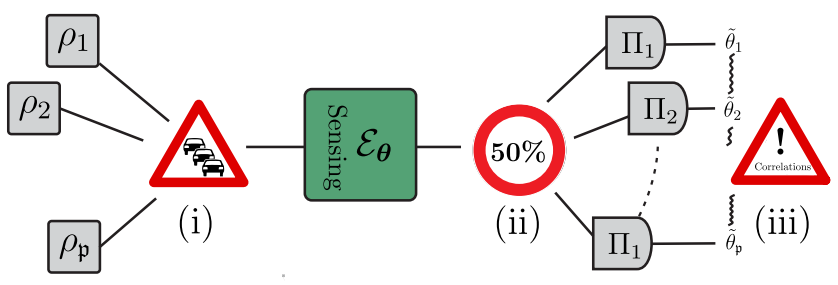

FIG. 1. Three potential sources of incompatibility in multiparameter quantum metrology. (i) Different input probe states may be optimal to estimate different parameters. This may in the worst-case lead to a $\mathfrak{p}$-fold increase in the resources consumed, compared with the best case where a single-probe state is optimal for estimating all parameters. (ii) Different incompatible measurements may be optimal for extracting information about different parameters. In generic noisy metrological models, this will require at most double the required resources in the asymptotic regime, where many probes may be used. (iii) If the chosen parametrization is not "natural" for the problem at hand, the resulting estimators will manifest correlations, and the imperfect knowledge of some parameters will have an impact on the effective estimation uncertainty of the remaining ones. are the main focus of this paper, the maximal quantum enhancement amounts to a constant factor gain, and the optimal probe states may be effectively approximated by product states of finitely entangled groups of particles [52,53]. As such, the argument on the impact of measurement incompatibility being at most a factor of 2 applies also to the asymptotically optimal strategies for noisy metrology.

Out of the compatibility conditions (i)-(iii) stated above, the most relevant one is actually condition (i) being the only one responsible for a different scaling of the asymptotic precision with the number of parameters involved, when comparing optimal protocols for simultaneous estimation with separate ones. We refer to the violation of condition (i) as probe incompatibility (for convenience, we keep the same name also when considering more general probing strategies with multiple uses of the channel), and it is the main focus of our analysis. When it comes to condition (iii), statistical independence of parameters can always be assured by a proper reparametrization; hence, provided one starts with a natural parametrization for a given model, this aspect of incompatibility can be avoided.

In this paper, we focus primarily on generic noisy channels, where the noise cannot be completely removed without hindering the parameter-encoding process. In this case, the asymptotic precision follows the so-called standard quantum limit (SQL) and quantum-enhanced strategies provide at most a constant gain [1,2]. Such noisy models, while generic and ubiquitous in practice, are much less studied in the multiparameter literature. A few particular instances have been examined, e.g., Refs. [54-59], but theoretical tools to identify fundamental bounds without neglecting the multiparameter character of the problem, especially probe incompatibility, are missing. We aim to close this gap.

\section{A. Summary of results}

First, in Sec. II we set the stage by defining a new figure of merit to quantify incompatibility in multiparameter quantum metrology. While this figure of merit takes into account all conditions (i)-(iii) (see Fig. 1), in the rest of the paper we focus on lower bounds that consider only condition (i), probe incompatibility.

To this end, in Sec. III we derive a new class of multiparameter precision bounds that hold for the most general adaptive strategy depicted in Fig. 2(a), extending previous single-parameter results $[1-3,60]$. In particular, we derive bounds that apply to various scenarios, as summarized in Table I. These represent our main technical contribution and, conveniently, the optimal bounds in this class can be evaluated with semidefinite programs presented in the Appendix F.

The idea behind the derivation is simple but powerful, and for a single-parameter it has proven to be the most powerful and widely applicable approach. A noisy channel can always be purified (formally) as a unitary interaction 
(a) Adaptive multiparameter estimation protocol

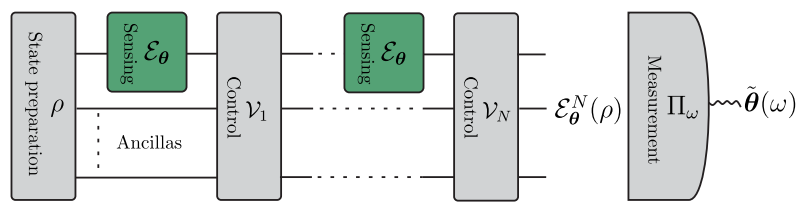

(b) random sensing scenario

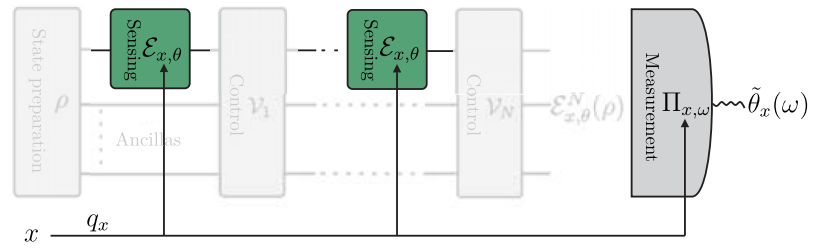

(c) Channel discrimination scenario

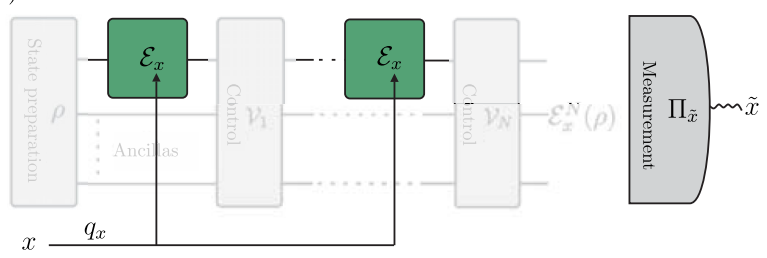

FIG. 2. Schematic representation of the three operational tasks considered in this paper. (a) Estimation of the parameters $\boldsymbol{\theta}=$ $\left(\theta_{1}, \ldots, \theta_{\mathfrak{p}}\right)$ appearing in the quantum channel $\mathcal{E}_{\boldsymbol{\theta}}$ under the most general entanglement-assisted adaptive strategy with $N$ uses of the channel. (b) Random quantum sensing, where a single parameter $\theta$ is encoded by a channel $\mathcal{E}_{x, \theta}$ randomly chosen from $\mathfrak{p}$ channels with probability $q_{x}$, and the extracted $x$ is revealed only before the measurement stage. (c) Channel discrimination under the most general strategy with $N$ uses of the channel.

between the system and an inaccessible environment. By choosing purifications that contain as little information as possible about the parameters, one can obtain tight bounds on the metrological precision. Thanks to a few technical adjustments, existing single-parameter derivations can be extended to multiple parameters in a way that takes into account probe incompatibility. Actually, these bounds are more general and apply also to a scenario that we call random quantum sensing depicted in Fig. 2(b) in which different channels chosen at random must be probed by the same state.

After introducing these tools, we then apply them to study probe incompatibility for a few paradigmatic models of noisy multiparameter quantum metrology in Sec. IV.

TABLE I. Summary of the bounds on the total QFI obtained in this paper. The SDPs can be found in Appendix F.

\begin{tabular}{lcc}
\hline \hline Case & Equation & SDP \\
\hline Single-channel use $(N=1)$ & $(12)$ & Yes \\
Parallel strategy, finite $N$ & $(\mathrm{C} 2)$ & Yes \\
Adaptive strategy, finite $N$ & $(19)$ & No \\
Parallel and adaptive, asymp. SQL & $(20)$ & Yes \\
Markovian noise, adaptive, asymp. SQL (in $T)$ & (D5) & Yes \\
\hline \hline
\end{tabular}

Finally, in Sec. V we show how multiparameter metrological bounds can be used to assess the ultimate performance of adaptive strategies in the task of discriminating between multiple quantum channels, sketched in Fig. 2(c). This approach allows us to close a conjecture presented in Ref. [61] and show that the quantum-computational speedup of the Grover search is ruined by dephasing and erasure noise.

\section{Relation to previous works}

A multiparameter bound for noisy channels and adaptive strategies was recently obtained without relying on purification arguments [62]. Despite being simple to evaluate, it is both less general and less tight than the optimal purification-based bounds we introduce here. Indeed, in Appendix E 2 we show that the class of bounds we introduce includes also the one of Ref. [62], which is generally suboptimal and might not give complete insight into probe incompatibility. Moreover, a purification-based methodology was introduced in Ref. [63], yet by construction it does not take into account probe incompatibility, since it requires performing a different convex optimization for each parameter. Finally, multiparameter bounds for paradigmatic models in optical metrology with losses have been obtained by purifying the dynamics $[54,55]$ but without considering the possibility of adaptive strategies.

\section{MULTIPARAMETER QUANTUM ESTIMATION}

\section{A. Quantum Fisher information matrix}

We consider a vector $\boldsymbol{\theta}=\left[\theta_{1}, \ldots, \theta_{\mathfrak{p}}\right]^{T}$ of $\mathfrak{p}$ real parameters that are encoded on a quantum state $\rho_{\boldsymbol{\theta}}=\mathcal{E}_{\boldsymbol{\theta}}(\rho)$ via the parameter-dependent quantum channel $\mathcal{E}_{\boldsymbol{\theta}}$ acting on the initial state $\rho$. In this work, we consider only finitedimensional systems.

A generic measurement is described by a positiveoperator-valued measure (POVM), i.e., a set of positive operators $\Pi_{\omega} \geq 0$ satisfying $\sum_{\omega} \Pi_{\omega}=\mathbb{1}$. We take the set of possible outcomes $\omega$ to be finite dimensional without loss of generality. The parameter-dependent classical probability distribution is obtained from the Born rule $p_{\boldsymbol{\theta}}(\omega)=\operatorname{Tr}\left[\Pi_{\omega} \rho_{\boldsymbol{\theta}}\right]$.

An estimator $\tilde{\boldsymbol{\theta}}(\omega)$ is a function that maps the random outcomes to estimated parameters values. The precision of the estimator is quantified by the mean-square-error matrix:

$$
\Sigma_{\tilde{\boldsymbol{\theta}}}:=\sum_{\omega} p_{\boldsymbol{\theta}}(\omega)[\tilde{\boldsymbol{\theta}}(\omega)-\boldsymbol{\theta}][\tilde{\boldsymbol{\theta}}(\omega)-\boldsymbol{\theta}]^{T} .
$$

In particular, we consider locally unbiased estimators that satisfy $\sum_{x} p_{\boldsymbol{\theta}^{*}}(\omega) \tilde{\boldsymbol{\theta}}(\omega)=\boldsymbol{\theta}^{*}$ and $\left.\sum_{\omega} \partial_{\theta_{i}} p_{\boldsymbol{\theta}}(\omega)\right|_{\boldsymbol{\theta}=\boldsymbol{\theta}^{*}} \times$ $\tilde{\theta}_{j}(\omega)=\delta_{i j}$; i.e., they are unbiased locally around the true value $\boldsymbol{\theta}^{*}$ of the parameter vector. In the following, we implicitly take all the derivatives with respect to the 
parameters $\theta_{j}$ evaluated at the true value and for simplicty of notation we write $\boldsymbol{\theta}$ instead of $\boldsymbol{\theta}^{*}$. For this class of estimators, the mean-square-error matrix is equal to the covariance matrix, and for any POVM, it satisfies the quantum Cramér-Rao bound (QCRB) [30-33]

$$
\Sigma_{\tilde{\boldsymbol{\theta}}} \geq \mathcal{F}^{-1}\left(\rho_{\boldsymbol{\theta}}\right),
$$

i.e., $\Sigma_{\tilde{\boldsymbol{\theta}}}-\mathcal{F}^{-1}\left(\rho_{\boldsymbol{\theta}}\right)$ is positive semidefinite, where we introduce the quantum Fisher information (QFI) matrix

$$
\mathcal{F}_{i j}\left(\rho_{\boldsymbol{\theta}}\right):=\operatorname{Re}\left(\operatorname{Tr}\left[\rho_{\boldsymbol{\theta}} L_{i} L_{j}\right]\right)
$$

written in terms of the symmetric logarithmic derivatives (SLDs) defined by the equations

$$
\partial_{\theta_{i}} \rho_{\boldsymbol{\theta}}=\frac{1}{2}\left(L_{i} \rho_{\boldsymbol{\theta}}+\rho_{\boldsymbol{\theta}} L_{i}\right) .
$$

For single-parameter problems $(\mathfrak{p}=1)$, it is always possible to find a POVM and a locally unbiased estimator to attain the QCRB (formally, at a given operation point $\theta^{*}$, or, more practically, uniformly in the asymptotic limit [64]). A possible choice for the optimal measurement is a projective measurement on the eigenbasis of the SLD operator. The multiparameter matrix bound (2), however, is not always attainable due to the possible noncommutativity of the SLDs $[31,34,64]$.

\section{B. Lower bounds on the total variance}

In an experiment that aims to estimate multiple parameters, in general there exists no strategy yielding a minimal covariance matrix [65]. A common choice is to quantify the overall error with a scalar, which we call the total variance

$$
\Delta_{W}^{2} \tilde{\boldsymbol{\theta}}:=\operatorname{tr}\left[W \Sigma_{\tilde{\boldsymbol{\theta}}}\right]
$$

where $W>0$ is a positive cost matrix (when $W=\mathbb{1}_{\mathfrak{p}}$ we simply write $\Delta^{2} \tilde{\boldsymbol{\theta}}$ ). Every cost matrix can be decomposed as $W=\sum_{k=1}^{\mathfrak{p}} w_{k} \mathbf{e}_{k} \mathbf{e}_{k}^{T}$, where $\mathbf{e}_{k}$ are orthonormal vectors and $w_{k}>0$ are strictly positive weights. A choice of cost matrix distinguishes a particular set of parameters associated with the eigenvectors of $W$ for which the estimation cost is determined by the corresponding eigenvalues $w_{k}$. In a sense, the cost matrix determines which parameters we regard as "separate." Without loss of generality, in what follows we assume to be working in the parametrization induced by $W$, so we need only to take into account the eigenvalues $w_{k}$.

From the matrix QCRB (2), we can lower bound the total variance as

$\Delta_{W}^{2} \tilde{\boldsymbol{\theta}} \geq \sum_{x=1}^{\mathfrak{p}} w_{x}\left[\mathcal{F}^{-1}\right]_{x x} \geq \sum_{x=1}^{\mathfrak{p}} \frac{w_{x}}{\mathcal{F}_{x x}} \geq \frac{\mathfrak{p}^{2}}{\sum_{x=1}^{\mathfrak{p}} w_{x}^{-1} \mathcal{F}_{x x}}$, where we write the Fisher information matrix elements $\mathcal{F}_{x x}$ in the $W$ eigenbasis.

The first inequality $\Delta_{W}^{2} \tilde{\boldsymbol{\theta}} \geq \operatorname{tr} W \mathcal{F}^{-1}$ is generally not attainable due to measurement incompatibility. A more fundamental bound is the Holevo Cramér-Rao bound, which is asymptotically attainable [34,51]. However, the Holevo Cramér-Rao bound is at most $2 \operatorname{tr} W \mathcal{F}^{-1}$ [48]; therefore, the asymptotic effect of incompatibility on the total variance is at most a factor 2 . As we argue in the Introduction, in this paper we are not concerned about measurement incompatibility, and we consider only bounds obtained from the QFI matrix.

The second inequality in Eq. (6) is a property of positive matrices, and it means that statistical correlations among the parameters, i.e., a nondiagonal $\mathcal{F}$, increase the error on the $x$ th parameter with respect to the single-parameter QCRB $1 / \mathcal{F}_{x x}$, i.e., assuming all other parameters are known. This inequality will be tight, provided the parameter basis choice induced by the cost matrix $W$ coincides with the parameter basis in which the QFI matrix is diagonal (we refer to this as a natural parametrization). The third inequality is obtained from the Cauchy-Schwarz inequality (or equivalently, from the inequality between harmonic and arithmetic mean) and is saturated when the diagonal elements are all equal. In multiparameter quantum metrology, the weaker bound (6) has often been used to avoid computing the inverse and simplify calculations; see, e.g., Refs. [21,62,66,67].

\section{Total QFI and random quantum sensing}

The multiparameter bounds we have discussed so far are defined for a $\mathfrak{p}$-parameter quantum channel $\mathcal{E}_{\boldsymbol{\theta}}$ (locally around the true value $\boldsymbol{\theta}^{*}$ ). For the sake of greater generality and anticipating the application in quantum-channel discrimination problems in Sec. V, we define instead a figure of merit for $\mathfrak{p}$ different single-parameter quantum channels $\left\{\mathcal{E}_{x, \theta_{x}}\right\}_{x=1}^{\mathfrak{p}}$ as

$$
\mathfrak{F}_{\mathbf{q}}(\rho):=\sum_{x=1}^{\mathfrak{p}} q_{x} \mathcal{F}\left(\mathcal{E}_{x, \theta_{x}}(\rho)\right),
$$

which we call the total QFI; $q_{x}$ are arbitrary positive weights. Clearly, the weighted trace of the multiparameter QFI matrix in Eq. (6) is also the total QFI of the single-parameter channels $\mathcal{E}_{\theta_{x}}$ defined by fixing the other $\mathfrak{p}-1$ parameters of $\mathcal{E}_{\boldsymbol{\theta}}$ to the true value, with weights $q_{x}=w_{x}^{-1}$.

When the channels $\mathcal{E}_{x, \theta_{x}}$ do not originate from the same multiparameter channel, there is no clear operational link to multiparameter estimation. Instead, we relate the total QFI to an operational task that we call random quantum sensing, formally described as single-parameter estimation of the channel $\rho \mapsto \sum_{x=1}^{\mathfrak{p}} q_{x} \mathcal{E}_{x, \theta}(\rho) \otimes|x\rangle\langle x|$, where we also assume that $q_{x}$ are probabilities $\sum_{x=1}^{\mathfrak{p}} q_{x}=1$ and to have 
a classical register of orthonormal states at the output $\left\langle x \mid x^{\prime}\right\rangle=\delta_{x, x^{\prime}}$. For the general case of multiple uses of the channel, as shown in Fig. 2(b), the classical register is not available after each use but only at the end of the protocol; formally, we deal with output states of the form $\sum_{x=1}^{\mathfrak{p}} q_{x} \mathcal{E}_{x, \theta}^{N}(\rho) \otimes|x\rangle\langle x|$. For such "quantum-classical" channels, the single-parameter QFI is the average QFI of the different channels and thus corresponds to the total QFI with $\theta_{x}=\theta$, which is thus a tight bound on the precision without the additional saturability issues in Eq. (6) affecting the multiparameter setting.

More practically, we can think of random quantum sensing as follows. Alice sends a probe state $\rho$ through a $\theta$-dependent channel $\mathcal{E}_{x, \theta}$ selected randomly according to the $\theta$-independent probability $q_{x}$ from the ensemble of $\mathfrak{p}$ channels. Alice does not know which channel $x$ is selected and has to choose a unique state $\rho$ to send in all runs. At the other end of the channel, Bob knows both Alice's probe state $\rho$ and the random value $x$, so each time he can implement an optimal measurement and estimator for the parameter $\theta$. Alice's goal is to help Bob estimate $\theta$ as precisely as possible; therefore, she has to prepare a state that is sensitive to $\theta$ for all different channels according to their probability. The quantity $\mathfrak{F}_{\mathbf{q}}(\rho)$ quantifies the precision that Bob obtains in estimating $\theta$ when Alice chooses to send $\rho$. This task, though different, is reminiscent of the quantum random-access-codes protocols $[68,69]$ in a sense that the sender must prepare a quantum state so that the information is extracted optimally even though it is a priori not clear how the information will be encoded (random quantum sensing) or which bit of information will be read out (random-access codes).

Interestingly, in a recent study of the relation between quantum steering and metrology [70], a multiple generator steering scenario was considered, which according to our definition was in fact a random sensing scenario. Moreover, the figure of merit considered there was exactly the total QFI. This strengthens the statement on the relevance of the random sensing scenario and indicates another interesting field of application for the methods developed in this paper.

\section{Incompatibility measures}

Here we introduce a quantity that captures the incompatibility of a given multiparameter quantum-channel estimation problem. We aim at a quantity that is 1 whenever there are no incompatibility issues and increases to some maximum value when the optimal probe for estimating a given parameter gives no information on the remaining ones. Moreover, we would like the quantity not to depend on the choice of the cost matrix or on the particular parametrization. The following natural quantity, which we refer to as the incompatibility measure of a multiparameter quantum channel, satisfies these requirements:

$$
\mathfrak{J}^{*}\left(\mathcal{E}_{\boldsymbol{\theta}}\right):=\max _{\left\{\mathbf{w}_{x}\right\}}\left(\frac{\min _{\rho, \Pi, \tilde{\boldsymbol{\theta}}} \Delta_{W}^{2} \tilde{\boldsymbol{\theta}}}{\sum_{x} \min _{\rho_{x}} \mathbf{w}_{x}^{T} \mathcal{F}^{-1}\left(\mathcal{E}_{\boldsymbol{\theta}}\left(\rho_{x}\right)\right) \mathbf{w}_{x}}\right),
$$

where $\tilde{\boldsymbol{\theta}}$ are locally unbiased estimators (equivalently, we could define this quantity in terms of the classical Fisher information matrix associated with a POVM without explicit reference to estimators), and the arbitrary (not necessarily orthogonal) vectors $\mathbf{w}_{x}$ determine the effective cost matrix for the multiparamter estimation problem $W=\sum_{x} \mathbf{w}_{x} \mathbf{w}_{x}^{T}$. Vectors $\mathbf{w}_{x}$ may be understood as representing a certain scalar function of $\boldsymbol{\theta}$ to be estimated, corresponding to a choice of a rank-1 cost matrix $W_{x}=$ $\mathbf{w}_{x} \mathbf{w}_{x}^{T}$. For a given $\rho_{x}$, the quantity $\mathbf{w}_{x}^{T} \mathcal{F}^{-1}\left(\mathcal{E}_{\boldsymbol{\theta}}\left(\rho_{x}\right)\right) \mathbf{w}_{x}$ appearing in the denominator is an attainable bound on the minimal error of estimating the function when the remaining parameters are treated as nuisance parameters [48,71] (due to the effectively scalar nature of the estimation problem, measurement incompatibility does not affect attainability of the bound in this case).

Intuitively, the incompatibility measure captures the worst-case scenario where the ratio between the cost of estimating $\mathfrak{p}$ scalar functions simultaneously is the largest compared with the cost of estimating them separately with the optimal probes. Note that $\mathfrak{J}^{*}$ is parametrization independent, since for any reparametrization $\boldsymbol{\theta} \rightarrow A \boldsymbol{\theta}$ (where $A$ is some invertible matrix), we obtain the identical formula by the following change of the cost vectors $\mathbf{w}_{x} \rightarrow A^{T} \mathbf{w}_{x}$. Moreover, this quantity is indeed 1 when there is a single state $\rho$ that gives minimal cost for all $x$.

Admittedly, this quantity is challenging to compute, especially for multiparameter metrological models in the presence of noise. Therefore, in this paper we use an efficiently computable lower bound, the probe incompatibility measure based on the total QFI:

$\mathfrak{I}^{*}\left(\mathcal{E}_{\boldsymbol{\theta}}\right) \geq \mathfrak{J}\left(\mathcal{E}_{\boldsymbol{\theta}}\right):=\mathfrak{p}\left(\max _{\rho} \sum_{x=1}^{\mathfrak{p}} \frac{\mathcal{F}_{x x}\left(\mathcal{E}_{\boldsymbol{\theta}}(\rho)\right)}{\max _{\rho_{x}} \mathcal{F}_{x x}\left(\mathcal{E}_{\boldsymbol{\theta}}\left(\rho_{x}\right)\right)}\right)^{-1}$

where some natural parametrization is fixed; see Appendix A for the derivation of the bound. This way, we obtain a quantity that takes values in the range $1 \leq \mathfrak{J}\left(\mathcal{E}_{\boldsymbol{\theta}}\right) \leq \mathfrak{p}$, where again 1 indicates perfect compatibility and $\mathfrak{p}$ maximal incompatibility (when the best strategy is to estimate the parameters separately and the state that maximizes the QFI for a given $x$ yields zero QFI for all other $x$ ). In what follows, instead of $\max _{\rho_{x}} \mathcal{F}_{x x}\left(\mathcal{E}_{\boldsymbol{\theta}}\left(\rho_{x}\right)\right)$ we also write $\max _{\rho_{x}} \mathcal{F}\left(\mathcal{E}_{\theta_{x}}\left(\rho_{x}\right)\right)$ with a single scalar parameter $\theta_{x}$ to indicate that this quantity refers to essentially a single-parameter problem, where all other parameters can be regarded as perfectly known.

Unlike the original incompatibility measure defined in Eq. (8), the lower bound (9) is in general parametrization dependent (though it is invariant under rescaling of parameters). For the lower bound (9) to hold, one must choose a 
natural parametrization, such that the QFI matrices corresponding to the optimal states $\operatorname{argmax}_{\rho_{x}} \mathcal{F}_{x x}\left(\mathcal{E}_{\boldsymbol{\theta}}\left(\rho_{x}\right)\right)$ are diagonal. Nonetheless, we argue that $\mathfrak{\Im}\left(\mathcal{E}_{\boldsymbol{\theta}}\right)$ is meaningful to study on its own for any given parametrization. While $\mathfrak{\Im}^{*}\left(\mathcal{E}_{\boldsymbol{\theta}}\right)$ takes into account all conditions (i)-(iii) stated in the Introduction, the probe incompatibility measure $\mathfrak{\Im}\left(\mathcal{E}_{\boldsymbol{\theta}}\right)$ singles out the effect of (i), as the name suggests. In principle, one can check a posteriori if the QFI matrices of the optimal states are diagonal to gauge the "naturalness" of the chosen parametrization.

Finally, let us note that when the probe incompatibility measure is applied to the random sensing scenario, the quantity (9) is actually the true incompatibility cost and not a lower bound, as there is a clear distinction between the parameters of different channels, unlike in the genuine multiparameter scenario.

\section{BOUNDS ON THE TOTAL QFI}

In this section, we study the total QFI in the most general case, considering a collection of $\mathfrak{p}$ quantum channels, i.e., completely positive trace-preserving (CPTP) maps acting on the same input Hilbert space $\mathcal{E}_{x, \theta_{x}}(\rho)=\sum_{j=1}^{r_{x}} K_{x, \theta_{x}, j} \rho K_{x, \theta_{x}, j}^{\dagger}$, where the Kraus operators of each channel satisfy $\sum_{j}^{r_{x}} K_{x, \theta_{x}, j}^{\dagger} K_{x, \theta_{x}, j}=\mathbb{1}$. The channels are labeled by $x=1, \ldots, \mathfrak{p}$, and each one depends on a parameter $\theta_{x}$ and can have a different number of Kraus operators $r_{x}$ and a different output dimension. We also introduce the column vectors of Kraus operators $\mathbf{K}_{x}=\left[K_{x, \theta_{x}, 1}, \ldots, K_{x, \theta_{x}, r_{x}}\right]^{T}$, which allows us to write sums over Kraus operators more compactly, e.g., $\mathbf{K}_{x}^{\dagger} \mathbf{K}_{x}=\mathbb{1}$. To ease the notation, we usually suppress the label $x$ and identify the different channels only through their parameter $\theta_{x}$ as $\mathcal{E}_{\theta_{x}}$, while leaving the dependence on $\theta_{x}$ of the Kraus operators implied.

Depending on the physical context, the resulting bounds will have applications either in the standard multiparameter estimation setting or in the random sensing scenario. In the multiparameter case, $x$ labels only the different parameters of the same quantum channel; thus, there is no explicit channel dependence on $x$, i.e., $\mathbf{K}_{x}=\mathbf{K}$ and $r_{x}=r$, and the notation $\mathcal{E}_{\theta_{x}}$ means that we are considering the $x$ th singleparameter submodel, as we explain in Sec. II C. On the other hand, for random sensing there are multiple channels but only one parameter $\theta_{x}=\theta$, so the notation $\mathcal{E}_{\theta_{x}}$ becomes shorthand for $\mathcal{E}_{x, \theta}$.

In this section, we derive an attainable bound for a single use of the channel and an upper bound for generic strategies with $N$ uses of the channel. In Appendix E, we also show that some previously known results, including the bound of Ref. [62], can be obtained within our approach. For practical applications, it is crucial to note that all bounds we derive [the single-use bound $\mathfrak{F}_{\mathbf{q}}(12)$ and the asymptotic channel bound $\mathfrak{B}_{\mathbf{q}}(20)$ ] can be computed with semidefinite programs (SDPs) similar to their single-parameter counterparts [2,4,72]; see Appendix F. A MATLAB implementation can be found in Ref. [73]. A summary of all bounds derived in this paper is shown in Table I.

\section{A. Single use of the channel}

Here we focus on the total channel QFI, i.e., the optimal total QFI:

$$
\mathfrak{F}_{\mathbf{q}}:=\max _{\rho} \mathfrak{F}_{\mathbf{q}}(\rho)=\max _{\rho} \sum_{x=1}^{\mathfrak{p}} q_{x} \mathcal{F}\left(\mathcal{E}_{\theta_{x}}(\rho)\right) .
$$

One can also consider a more general entanglementassisted strategy corresponding to the extended channels $\mathcal{E}_{\theta_{x}} \otimes \mathcal{I}_{A}\left(\mathcal{I}_{A}\right.$ denotes the identity channel on an auxiliary Hilbert space) so that the maximization runs over bipartite states $\rho \in \mathcal{S}\left(\mathcal{H}_{S} \otimes \mathcal{H}_{A}\right)$, where $\mathcal{S}(\mathcal{H})$ denotes the space of density matrices over $\mathcal{H}$. The total channel QFI for this extended channel is always an upper bound on $\mathfrak{F}_{\mathbf{q}}$. In this work, we do not investigate the difference between assisted and unassisted strategies; therefore, the distinction between the two cases is not crucial and will not be made explicit unless necessary, even if the bounds are actually derived for the extended channels.

The most straightforward way to upper bound the total channel QFI is to use the single-parameter channel QFI $\mathfrak{F}_{x}$ for each parameter:

$$
\mathfrak{F}_{\mathbf{q}} \leq \sum_{x=1}^{\mathfrak{p}} q_{x} \max _{\rho_{x}} \mathcal{F}\left(\mathcal{E}_{\theta_{x}}\left(\rho_{x}\right)\right)=\sum_{x=1}^{\mathfrak{p}} q_{x} \mathfrak{F}_{x} .
$$

This upper bound, however, does not take into account potential probe incompatibility, and indeed, when it is attained the incompatibility cost takes its minimal value $\mathfrak{J}\left(\mathcal{E}_{\boldsymbol{\theta}}\right)=1$.

In order to obtain a tighter bound, we revisit and generalize the derivation of the single-parameter channel QFI ([60] Theorem 4), which results in the following.

Theorem 1: The total channel QFI of a collection of quantum channels is upper bounded as

$$
\mathfrak{F}_{\mathbf{q}} \leq 4 \min _{\mathfrak{h}}\left\|\sum_{x=1}^{\mathfrak{p}} q_{x} \alpha_{x}\right\|,
$$

where $\|\cdot\|$ denotes the operator norm (maximal singular value), $\alpha_{x}:=\partial_{\theta_{x}} \tilde{\mathbf{K}}_{x}^{\dagger} \partial_{\theta_{x}} \tilde{\mathbf{K}}_{x}$ with $\partial_{\theta_{x}} \tilde{\mathbf{K}}_{x}:=\partial_{\theta_{x}} \mathbf{K}_{x}-i h_{x} \mathbf{K}_{x}$. and $\mathfrak{h}=\left\{h_{x}\right\}_{x=1}^{\mathfrak{p}}$ is a collection of $\mathfrak{p}$ Hermitian matrices, each of dimension $r_{x} \times r_{x}$. Equality in Eq. (12) is attained when considering the extended channels $\mathcal{E}_{\theta_{x}} \otimes \mathcal{I}_{A}$.

Proof.-We consider the extended channels $\mathcal{E}_{\theta_{x}} \otimes \mathcal{I}_{A}$, which will give an upper bound for the unextended ones. First, we note that the total QFI is maximized by a pure state $|\psi\rangle \in \mathcal{H}_{S} \otimes \mathcal{H}_{A}$ because of its convexity. Each single- 
parameter QFI in the sum can be written as a minimization over a Hermitian matrix $h_{x}[2,60]$ :

$$
\begin{aligned}
\mathcal{F}\left(\mathcal{E}_{\theta_{x}} \otimes \mathcal{I}(|\psi\rangle)\right) & =4 \min _{h_{x}}\left\langle\psi\left|\partial_{\theta_{x}} \tilde{\mathbf{K}}_{x}^{\dagger} \partial_{\theta_{x}} \tilde{\mathbf{K}}_{x} \otimes \mathbb{1}\right| \psi\right\rangle \\
& =4 \min _{h_{x}} \operatorname{Tr}\left[\rho \partial_{\theta_{x}} \tilde{\mathbf{K}}_{x}^{\dagger} \partial_{\theta_{x}} \tilde{\mathbf{K}}_{x}\right],
\end{aligned}
$$

where $\rho=\operatorname{Tr}_{A}|\psi\rangle\langle\psi|$, and we use shorthand notation $\mathcal{E}(|\psi\rangle) \equiv \mathcal{E}(|\psi\rangle\langle\psi|)$. Therefore, it is equivalent to maximize the lhs of Eq. (13) over pure bipartite states and the rhs over the convex set of mixed states $\mathcal{S}(\mathcal{H})$. The minimum of the sum of functions of the independent variables $h_{x}$ is equal to the sum of the minima; thus, we obtain

$$
\begin{gathered}
\max _{|\psi\rangle} \sum_{x=1}^{\mathfrak{p}} q_{x} \mathcal{F}\left(\mathcal{E}_{\theta_{x}} \otimes \mathcal{I}(|\psi\rangle)\right) \\
=4 \max _{\rho \in \mathcal{S}(\mathcal{H})} \min _{\mathfrak{h}} \operatorname{Tr}\left[\rho \sum_{x=1}^{\mathfrak{p}} q_{x} \partial_{\theta_{x}} \tilde{\mathbf{K}}_{x}^{\dagger} \partial_{\theta_{x}} \tilde{\mathbf{K}}_{x}\right] \\
=4 \min _{\mathfrak{h}} \max _{\rho \in \mathcal{S}(\mathcal{H})} \operatorname{Tr}\left[\rho \sum_{x=1}^{\mathfrak{p}} q_{x} \partial_{\theta_{x}} \tilde{\mathbf{K}}_{x}^{\dagger} \partial_{\theta_{x}} \tilde{\mathbf{K}}_{x}\right] \\
=4 \min _{\mathfrak{h}}\left\|\sum_{x=1}^{\mathfrak{p}} q_{x} \partial_{\theta_{x}} \tilde{\mathbf{K}}_{x}^{\dagger} \partial_{\theta_{x}} \tilde{\mathbf{K}}_{x}\right\| .
\end{gathered}
$$

The conditions to interchange max and $\min$ in Eq. (15) are the convexity and compactness of the set $\mathcal{S}(\mathcal{H})$, the concavity of the cost function in $\rho$, and its convexity in the variables $h_{x}$ ([74] Corollary 37.3.2). These conditions are satisfied since the function is linear in $\rho$, each term in the sum is convex in the matrix $h_{x}$, and the sum of convex functions with positive coefficients is convex.
Since the bound (12) is the norm of a sum of operators, Eq. (11) immediately follows from the triangle inequality, where $\mathfrak{F}_{x}=4 \min _{h_{x}}\left\|\partial_{\theta_{x}} \tilde{\mathbf{K}}_{x}^{\dagger} \partial_{\theta_{x}} \tilde{\mathbf{K}}_{x}\right\|$ is the single-parameter extended channel QFI [60]. The bound (12) with weights $q_{x}=1 / \mathfrak{F}_{x}$ is used to evaluate the probe incompatibility cost (9) for the (extended) channel. Finally, as a consistency check, we can see that for factorized channels of the form $\mathcal{E}_{1, \theta_{1}} \otimes \cdots \otimes \mathcal{E}_{\mathfrak{p}, \theta_{\mathfrak{p}}}$ where each parameter is encoded independently from the others, the triangle inequality is always saturated because each $\alpha_{x}$ acts nontrivially only on a single subsystem and thus, $\left\|\sum_{x} \alpha_{x}\right\|=$ $\left\|\sum_{x} \mathbb{\otimes} \ldots \otimes \partial_{x} \mathbf{K}_{x}^{\dagger} \partial_{x} \mathbf{K}_{x} \ldots \otimes \mathbb{1}\right\|=\sum_{x}\left\|\partial_{x} \mathbf{K}_{x}^{\dagger} \partial_{x} \mathbf{K}_{x}\right\|$.

\section{B. Asymptotic bound for the most general strategy}

Now we move to a metrological strategy with $N$ uses of the channel $\mathcal{E}_{\theta_{x}}$. We consider the most general adaptive strategy, allowing for arbitrary auxiliary systems and unitary control operations $\mathcal{V}_{i}(\rho)=V_{i} \rho V_{i}^{\dagger}$, as depicted in Fig. 2(a). The overall channel is thus, $\mathcal{E}_{\theta_{x}}^{N}=\mathcal{V}_{N^{\circ}}\left(\mathcal{E}_{\theta_{x}} \otimes\right.$ $\mathcal{I}$ ) $\circ \mathcal{V}_{N-1} \circ \ldots \circ \mathcal{V}_{1} \circ\left(\mathcal{E}_{\theta_{x}} \otimes \mathcal{I}\right.$ ) (when the input and output dimensions of the channels are different, unitaries and auxiliary systems are used to make them compatible), and we want to upper bound the total QFI of the sequential scheme

$$
\mathfrak{F}_{\mathbf{q}}^{N}:=\max _{\rho,\left\{\mathcal{V}_{i}\right\}} \sum_{x=1}^{\mathfrak{p}} q_{x} \mathcal{F}\left(\mathcal{E}_{\theta_{x}}^{N}(\rho)\right) .
$$

We stress that the optimal strategy, i.e., not only the initial state but also the control unitaries, cannot use any information about which channel $x$ is applied $N$ times. The main result for the general strategy is the following.

Theorem 2: The total channel QFI for an adaptive strategy with $N$ uses of the channel satisfies the bound

$$
\mathfrak{F}_{\mathbf{q}}^{N} \leq 4 \min _{\mathfrak{h}}\left\{N\left\|\sum_{x} q_{x} \alpha_{x}\right\|+N(N-1) \max _{x}\left(\left\|\beta_{x}\right\|\right)\left[\left\|\sum_{x=1}^{\mathfrak{p}} q_{x} \beta_{x}\right\|+2 \sqrt{\left.\left(\sum_{x=1}^{\mathfrak{p}} q_{x}\right)\left\|\sum_{x=1}^{\mathfrak{p}} q_{x} \alpha_{x}\right\|\right]}\right\}\right.
$$

where $\quad \alpha_{x}=\left(\partial_{\theta_{x}} \mathbf{K}_{x}-i h_{x} \mathbf{K}_{x}\right)^{\dagger}\left(\partial_{\theta_{x}} \mathbf{K}_{x}-i h_{x} \mathbf{K}_{x}\right) \quad$ and $\beta_{x}=\left(\partial_{\theta_{x}} \mathbf{K}_{x}-i h_{x} \mathbf{K}_{x}\right)^{\dagger} \mathbf{K}_{x}$.

The proof is relegated to Appendix B. For $\mathfrak{p}=1$, one obtains the known single-parameter bound $[3,8,75]$.

The parallel strategy corresponding to the channel $\mathcal{E}_{\theta_{x}}^{\otimes N}$ is less powerful than a sequential one since it can be obtained by choosing swap operations as the control unitaries. For completeness, we provide a complete derivation of the tighter bound for the parallel strategy in Appendix C. Note that the two bounds differ asymptotically only when Heisenberg scaling is allowed. However, since we are mostly interested in noisy channels that satisfy the conditions $\beta_{x}=0 \forall x=1, \ldots, \mathfrak{p}$, this distinction will not be relevant. In this case, the optimal upper bound in Eq. (19) is asymptotically linear in $N$ :

$$
\mathfrak{F}_{\mathbf{q}}^{N} \leq N \mathfrak{B}_{\mathbf{q}}, \quad \mathfrak{B}_{\mathbf{q}}:=4 \min _{\mathfrak{h}, \beta_{x}=0}\left\|\sum_{x=1}^{\mathfrak{p}} q_{x} \alpha_{x}\right\|
$$

We call $\mathfrak{B}_{\mathbf{q}}$ the asymptotic SQL bound, since in the limit $N \gg 1$ the optimal variables $h_{x}$ in Eq. (19) must make the quadratic term vanish. Furthermore, in Appendix D, we provide a time-continuous variant of the bound that applies to general Markovian noise, where the duration of a single 
probing step may be adjusted arbitrarily. In this case, it is the total interrogation time that is treated as a resource, and the bound is a direct generalization of the single-parameter bounds derived in Refs. [4,5].

The condition $\beta_{x}=0$ is dubbed the "Hamiltonian in the Kraus span" (HKS) condition:

$$
\beta_{x}=0 \Leftrightarrow i \partial_{\theta_{x}} \mathbf{K}_{x}^{\dagger} \mathbf{K}_{x} \in \operatorname{span}_{\mathbb{R}}\left(K_{x, i}^{\dagger} K_{x, j}, \quad \forall i, j\right),
$$

where we introduce the so-called Kraus spans [8] of the channels $\mathcal{E}_{\theta_{x}}$. Here, $i \partial_{\theta_{x}} \mathbf{K}_{x}^{\dagger} \mathbf{K}_{x}$ is not a real Hamiltonian but an effective generator for the parameter $\theta_{x}$. In the simple case of parameter-independent noise following a singleparameter unitary, we have the Kraus operators $K_{j} e^{-i \theta H}$ and indeed $H=i \partial_{\theta} \mathbf{K}^{\dagger} \mathbf{K}$. For an arbitrary parameter of a full-rank channel, this condition is always satisfied, thus ruling out Heisenberg scaling $N^{2}$ for almost all (in a measure-theoretical sense) quantum channels [60].

Just like in the single-use case, the sum of singleparameter bounds is an upper bound that does not take into account probe incompatibility and the triangle inequality implies $\mathfrak{B}_{\mathbf{q}} \leq \sum_{x=1}^{\mathfrak{p}} q_{x} \mathfrak{B}_{\theta_{x}}$, where $\mathfrak{B}_{\theta_{x}}:=4 \min _{h_{x}}\left\|\alpha_{x}\right\|$ subject to $\beta_{x}=0$. Following this observation, we introduce an asymptotic probe incompatibility measure that generalizes Eq. (9). In the SQL case, it is obtained by evaluating $\mathfrak{B}_{\mathbf{q}}$ with weights $q_{x}=1 / \mathfrak{B}_{\theta_{x}}$ :

$$
\mathfrak{J}_{\infty}\left(\mathcal{E}_{\boldsymbol{\theta}}\right):=\mathfrak{p}\left(\underset{\substack{\left.\hat{\mathfrak{h}} \mathfrak{h}=0\} \\ \min _{x}=0\right\}}}{\|}\left\|\sum_{x=1}^{\mathfrak{p}} \frac{\alpha_{x}}{\mathfrak{B}_{\theta_{x}}}\right\|\right)^{-1} .
$$

Since the single-parameter bounds $\mathfrak{B}_{\theta_{x}}$ are asymptotically attainable [8], the quantity $\mathfrak{J}_{\infty}$ is a computable lower bound on the actual asymptotic incompatibility.

\section{Purification-based definition of the QFI matrix}

In the proof of Theorem 1, we use the established purification-based definition of the single-parameter QFI. Since we work assuming that each channel $\mathcal{E}_{\theta_{x}}$ can be different, the purifications pertaining to different parameters need not be related. However, in the case of multiple parameters and a single channel, the need to choose a different purification for each parameter would entail that the bound is not tight. In other words, the upper bound (12) would not necessarily correspond to the weighted trace of the QFI matrix of the optimal probe state on which the channel acted upon.

In this section, we show that this is not the case and that the purification-based definition of the scalar QFI is easily generalized to the matrix-valued case (a similar statement appeared in Ref. [55] without explicit proof), meaning that each choice of the matrices $\mathfrak{h}$ corresponds to a unique purification.
For notational convenience, we introduce the $d \times \mathfrak{p}$ Jacobian matrix of a $d$-dimensional pure state $\left|\Psi_{\boldsymbol{\theta}}\right\rangle: \nabla \Psi_{\boldsymbol{\theta}}=$ $\left[\left|\partial_{1} \Psi_{\theta}\right\rangle, \ldots,\left|\partial_{\mathfrak{p}} \Psi_{\boldsymbol{\theta}}\right\rangle\right]$ so that we can write the $\mathfrak{p} \times \mathfrak{p}$ QFI matrix compactly as

$$
\mathcal{F}\left(\left|\Psi_{\theta}\right\rangle\right)=4 \operatorname{Re}\left[\left(\nabla \Psi_{\theta}\right)^{\dagger} \nabla \Psi_{\theta}-\left(\nabla \Psi_{\theta}\right)^{\dagger}\left|\Psi_{\theta}\right\rangle\left\langle\Psi_{\theta}\right| \nabla \Psi_{\theta}\right],
$$

and clearly $\mathcal{F}\left(\left|\Psi_{\theta}\right\rangle\right) \leq 4 \operatorname{Re}\left[\left(\nabla \Psi_{\theta}\right)^{\dagger} \nabla \Psi_{\theta}\right]$ since the term subtracted is a positive-semidefinite matrix. We can thus formulate the result as follows.

Theorem 3: The QFI matrix of a mixed state $\rho_{\boldsymbol{\theta}}$ is equal to the minimal (in the positive-semidefinite sense) QFI matrix of its purifications

$$
\mathcal{F}\left(\rho_{\boldsymbol{\theta}}\right)=\min _{\Psi_{\boldsymbol{\theta}}} \mathcal{F}\left(\left|\Psi_{\boldsymbol{\theta}}\right\rangle\right)=4 \min _{\Psi_{\boldsymbol{\theta}}} \operatorname{Re}\left[\left(\boldsymbol{\nabla} \Psi_{\boldsymbol{\theta}}\right)^{\dagger} \boldsymbol{\nabla} \Psi_{\boldsymbol{\theta}}\right] .
$$

Proof.-Starting from an arbitrary fixed purification $\left|\Psi_{\boldsymbol{\theta}}\right\rangle$, such that $\rho_{\boldsymbol{\theta}}=\operatorname{Tr}_{E}\left|\Psi_{\boldsymbol{\theta}}\right\rangle\left\langle\Psi_{\boldsymbol{\theta}}\right|$, all other purifications of the quantum statistical model are obtained by acting with a parameter-dependent unitary on the environment $\left|\tilde{\Psi}_{\theta}\right\rangle=\mathbb{1} \otimes u_{\theta}\left|\Psi_{\theta}\right\rangle$. If we consider the matrix $\left(\nabla \tilde{\Psi}_{\theta}\right)^{\dagger} \nabla \tilde{\Psi}_{\theta}$, we see that the unitary $u_{\theta}$ enters only through the quantities $u_{\theta}^{\dagger} \partial_{j} u_{\theta}=-i h_{j}$, where $h_{j}$ are Hermitian matrices. Crucially, the matrices $h_{j}$ are independent variables [76]. From the purification-based definition of the singleparameter QFI [60,72] $\mathcal{F}_{j j}\left(\rho_{\boldsymbol{\theta}}\right)=\min _{h_{j}}\left\langle\partial_{j} \Psi_{\boldsymbol{\theta}} \mid \partial_{j} \Psi_{\boldsymbol{\theta}}\right\rangle=$ $\mathcal{F}_{j j}\left(\left|\Psi_{\boldsymbol{\theta}}^{*}\right\rangle\right)$, we know that for each parameter there exists an optimal matrix $h_{j}$ such that the pure state model $\left|\Psi_{\boldsymbol{\theta}}^{*}\right\rangle$ satisfies $\left|\partial_{j} \Psi_{\boldsymbol{\theta}}^{*}\right\rangle=\frac{1}{2} L_{j} \otimes \mathbb{1}\left|\Psi_{\boldsymbol{\theta}}^{*}\right\rangle$, where $L_{j}$ is the SLD of the original mixed state $[60,77]$, also implying $\left\langle\partial_{j} \Psi_{\theta}^{*} \mid \Psi_{\theta}^{*}\right\rangle=0$. Therefore, for this purification we obtain the equality

$$
\operatorname{Tr}\left[\rho_{\boldsymbol{\theta}} L_{i} L_{j}\right]=4\left\langle\partial_{i} \Psi_{\boldsymbol{\theta}}^{*} \mid \partial_{j} \Psi_{\boldsymbol{\theta}}^{*}\right\rangle \Rightarrow \mathcal{F}\left(\rho_{\boldsymbol{\theta}}\right)=\mathcal{F}\left(\left|\tilde{\Psi}_{\boldsymbol{\theta}}\right\rangle\right),
$$

where the QFI matrix is the real part of the complex matrix on the left. Finally, thanks to the monotonicity of the QFI matrix [33,78], we have the matrix inequalities

$$
\mathcal{F}\left(\rho_{\boldsymbol{\theta}}\right) \leq \mathcal{F}\left(\left|\tilde{\Psi}_{\boldsymbol{\theta}}\right\rangle\right) \leq 4 \operatorname{Re}\left[\left(\boldsymbol{\nabla} \tilde{\Psi}_{\boldsymbol{\theta}}\right)^{\dagger} \nabla \tilde{\Psi}_{\boldsymbol{\theta}}\right]
$$

which hold for arbitrary purifications $\left|\tilde{\Psi}_{\theta}\right\rangle$ since partial tracing is a CPTP map. This shows that the matrix-valued minimization in the statement of the theorem is well posed and closes the proof.

We remark that when $\operatorname{Im}\left(\operatorname{Tr}\left[\rho_{\theta} L_{i} L_{j}\right]\right)=0 \forall i, j$, the bound $\operatorname{Tr}\left[W \mathcal{F}\left(\rho_{\boldsymbol{\theta}}\right)^{-1}\right]$ is equal to the Holevo Cramér-Rao bound and thus asymptotically attainable [29,79]. Therefore, thanks to the equality between the complex matrices (25), this condition can be checked from the optimal purification.

By solving the minimization (12), one obtains the maximal value of the trace of the QFI matrix, but the 
optimal state is not identified and it is not immediate to retrieve the full QFI matrix. In Appendix G, we present an algorithm that finds an optimal state by enforcing that a solution of the minimax problem in Eq. (15) must be a saddle point in the variables $\rho$ and $\mathfrak{h}$, analogous to the single-parameter case [8]. The same approach can be applied to the asymptotic SQL bound (20), but the meaning of the obtained $\rho$ is unclear, unlike for single-parameter problems where it is connected with the approximate error correction strategy to attain the bound [8]. Once an optimal state is found, it is easy to obtain its full QFI matrix using the purification-based definition.

\section{APPLICATIONS TO MULTIPARAMETER QUANTUM METROLOGY}

In this section, we apply the theory we developed to physical problems schematized in Fig. 3. First, we study the paradigmatic problem of characterizing the Hamiltonian of a $d$-dimensional quantum system, i.e., Hamiltonian tomography, in which the number of parameters scales quadratically with the dimension of the Hilbert space. We consider the effect of erasure noise, and we show that asymptotic probe incompatibility is identical to the noiseless case. Focusing on the submodel with only commuting generators, the problem is equivalent to multiphase estimation in the presence of optical losses, a widely studied task for which we confirm and strengthen existing results. Then, we consider the estimation of multiple optical losses, for which our bound is tight. Finally, we focus on the estimation of a phase and a noise parameter for photon loss and phase diffusion. In these instances, we see no asymptotic probe incompatibility, matching physical intuition, numerical evidence, and previous results.
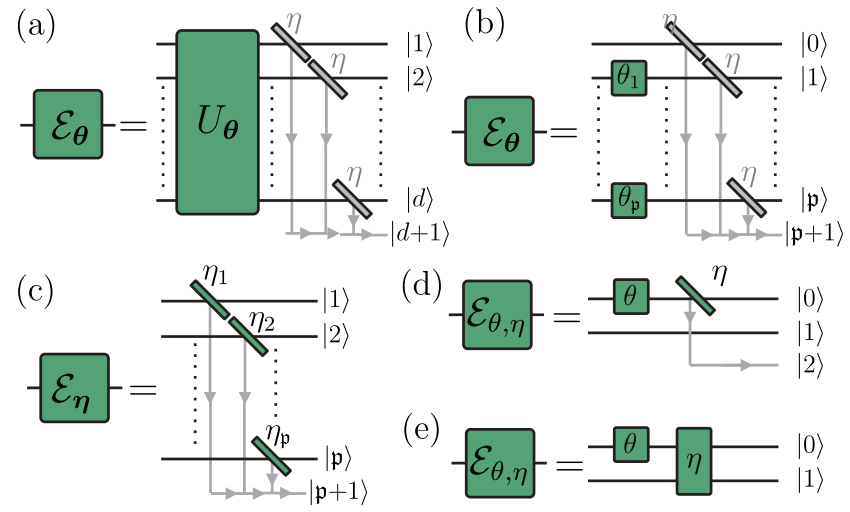

(e)

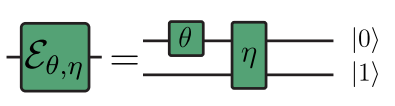

FIG. 3. Schematic representation of the tasks considered in this section: simultaneous estimation of (a) the parameters of an arbitrary $\mathrm{U}(d)$ transformation with erasure noise; (b) multiple phases in the presence of uniform loss on all modes; (c) multiples losses; (d) phase and loss imprinted by a sample in a two-arm interferometer; (e) phase and dephasing in a two-arm interferometer.
An additional example, the estimation of the two noise parameters of a generalized amplitude damping channel, is studied in Appendix H 1. For this channel, as well as for the multiple-loss channel, there is no advantage in using advanced strategies, since the asymptotic SQL bound coincides with the single-use one, analogous to several single-parameter noise estimation problems with qubits [72].

\section{A. Hamiltonian tomography with erasure noise}

Before focusing on this specific model, we start with a more general consideration for the estimation of the parameters $\boldsymbol{\theta}$ of a unitary $U_{\boldsymbol{\theta}}=e^{-i \sum_{x}^{\mathfrak{p}} \theta_{x} G_{x}}$ with the Hermitian generators $G_{x}$, when the noisy channel acts after the unitary encoding. The Kraus operators are thus, $K_{i}^{\prime}=K_{i} U_{\theta}$, where $K_{i}$ is the Kraus operator of the noise only, and its derivatives are $\partial_{x} K_{i}^{\prime}=-i K_{i}^{\prime} G_{x}$ since we assume to work at the true value $\theta_{x}=0 \forall x$. Under these assumptions, the SQL bound (20) can be simplified using the HKS conditions:

$\mathfrak{B}_{\mathbf{q}}=4 \min _{\substack{h_{x} \\ \beta^{x}=0}}\left\|-\left(\sum_{x=1}^{\mathfrak{p}} q_{x} G_{x}^{2}\right)+\mathbf{K}^{\dagger}\left(\sum_{x=1}^{\mathfrak{p}} q_{x} h_{x}^{2}\right) \mathbf{K}\right\|$.

Hamiltonian tomography of a $d$-dimensional quantum system amounts to the estimation of the $d^{2}-1$ parameters of a $\mathrm{SU}(d)$ transformation. This problem, or slight variations, has been studied in the noiseless and error-corrected scenario $[19,21,28,80-82]$. It has been shown that an advantage of $O(\sqrt{\mathfrak{p}})=O(d)$ is possible using a simultaneous adaptive estimation strategy.

Here, for simplicity we consider the $\mathfrak{p}=d^{2}$ parameters of a $\mathrm{U}(d)$ transformation. This choice makes the QFI matrix singular, but it is not a problem when using the total QFI as a figure of merit. Concretely, we choose the following generators:

$$
\begin{aligned}
G_{j}^{\mathrm{diag}} & =|j\rangle\langle j|, \quad G_{j k}^{\mathrm{re}}=\frac{1}{2}(|j\rangle\langle k|+| k\rangle\langle j|), \\
G_{j k}^{\mathrm{im}} & =\frac{i}{2}(|j\rangle\langle k|-| k\rangle\langle j|),
\end{aligned}
$$

where we separate the three submodels with $d$ diagonal, $d(d-1) / 2$ real, and $d(d-1) / 2$ imaginary off-diagonal generators. For the noise, we consider a qudit erasure channel, i.e., $\mathcal{E}(\rho)=\eta \rho+(1-\eta)|d+1\rangle\langle d+1|$, described by the following $d+1$ Kraus operators:

$$
K_{0}=\sqrt{\eta}\left[\begin{array}{c}
\mathbb{1}_{d} \\
0, \ldots, 0
\end{array}\right] \quad K_{i}=\sqrt{1-\eta}|d+1\rangle\langle i|
$$

for $i=1, \ldots, d$; the output Hilbert space contains an additional dimension that represents the system in the "lost" state $|d+1\rangle$, as shown in Fig. 3(a). 
The asymptotic SQL bound on the total QFI is the following (more details in Appendix H2):

$$
\begin{gathered}
\mathfrak{B}=\frac{\eta}{1-\eta}\left(\mathfrak{\mho}_{\text {diag }}+\mathfrak{F}_{\text {real }}+\mathfrak{\mho}_{\text {imag }}\right), \\
\mathfrak{F}_{\text {diag }}=\frac{4(d-1)}{d}, \quad \mathfrak{F}_{\text {real }}=\mathfrak{F}_{\text {imag }}=d-1,
\end{gathered}
$$

where the weights are $q_{i}=1$ (in this case, we drop the weight index), and $\mathfrak{F}$ are the noiseless bounds for a single use of the channel for the three submodels (attained by considering the extended channel and a probe state maximally entangled with the auxiliary system [19]). This means that probe incompatibility exists only inside the three submodels.

The single-parameter bounds are identical for all parameters: $\mathfrak{B}_{x}=[\eta /(1-\eta)]$, equivalent to the estimation of a qubit rotation with erasure noise [1-3]. This means that the asymptotic probe incompatibility cost is simply obtained by rescaling the bound (30), and it is identical to the noiseless case $\mathfrak{I}_{\infty}=\left\{d^{3} /\left[2\left(d^{2}+d-2\right)\right]\right\}=O(\sqrt{\mathfrak{p}})$, which is an intermediate scaling between the two extremal scalings $O(1)$ (compatibility) and $O(\mathfrak{p})$ (maximal incompatibility).

\section{B. Multiple phases with loss}

Estimating multiple optical phases simultaneously is a paradigmatic task in multiparameter quantum metrology $[12,20,22,66,83-88]$ also realized experimentally [24,89]. In the noiseless scenario, Humphreys et al. [12] argued that a simultaneous estimation strategy provides an advantage in the total variance over individual quantum estimation schemes that scales as $O(\mathfrak{p})$. However, the apparent advantage is actually a pitfall in the application of the QCRB. A minimax analysis taking into account the total number of photons shows that no advantage in scaling with $\mathfrak{p}$ is present [90]. Fortunately, asymptotic discrepancies between the minimax, Bayesian, and QCRB predictions may appear only in the Heisenberg scaling scenarios and disappear when the optimal scaling corresponds to the SQL, and this issue will not have any impact on our results.

Here we study the problem in the presence of optical losses that forbid Heisenberg scaling. This setting was first studied in Ref. [55] with a purification-based bound, and the problem was also studied treating the transmissivity as a nuisance parameter [51]. In previous works, lossy interferometers were modeled in a "second quantization" description where each mode is treated as a quantum subsystem. We take a different approach and consider photons as the elementary subsystems using a "first quantization" formalism which treats photons as formally distinguishable particles [91]. This can describe, e.g., photons prepared in nonoverlapping time bins. In this picture, each mode represents a dimension of the single- photon Hilbert space, and the fundamental object is thus the qudit channel describing the evolution of a single photon. While in practice the mode description is well suited to describe optical interferometers, from a formal and fundamental point of view it models only parallel strategies in which single-photon channels are probed in parallel by permutationally symmetric multiphoton states. By working in a more general scenario and treating the photons as distinguishable, we can investigate the possible usefulness of adaptive strategies. In this picture, the erasure channel (29) describes photon losses happening with equal probability $1-\eta$ in each mode.

Mathematically, this problem is described by a commuting submodel of the full $\mathrm{U}(d)$ model we introduced before. We consider only $\mathfrak{p}=d-1$ parameters corresponding to all diagonal generators in Eq. (28) except one representing the reference arm of the interferometer with a known phase, as shown in Fig. 3(b). Since the generators commute, the bound (27) now holds for all values of the parameters. We obtain the following bound on the total variance:

$$
\Delta^{2} \tilde{\boldsymbol{\theta}} \geq \frac{1-\eta}{\eta} \frac{\mathfrak{p}^{3}}{4 N(\mathfrak{p}-1)} \geq \frac{1-\eta}{4 \eta} \frac{\mathfrak{p}^{2}}{N}
$$

valid for $\mathfrak{p}>1(d>2)$, since the total QFI is identical to the bound (30) on diagonal parameters, with the substitution $d \rightarrow d-1$, for $d>2$. As we explain in more detail in Appendix $\mathrm{H} 2 \mathrm{a}$, when the same $\mathfrak{p}$ diagonal generators act on higher-dimensional systems, the optimal total QFI is unchanged. This means that adding additional reference modes does not improve the bound. The rightmost quantity in Eq. (32) corresponds to the bound obtained in Ref. [55] for simultaneous estimation of the $\mathfrak{p}$ phases with $N$ total indistinguishable photons in the limit $N \gg 1, \mathfrak{p} \gg 1$, $N / \mathfrak{p} \gg 1$. Our result shows that the same bound holds even for the most general adaptive strategy acting on distinguishable photons.

The asymptotic probe incompatibility cost is almost maximal $\mathfrak{\Im}_{\infty}=\left\{\mathfrak{p}^{2} /[4(\mathfrak{p}-1)]\right\}=O(\mathfrak{p})$, and it is again identical to the noiseless case. This means that little can be gained from simultaneous estimation. Indeed, the asymptotic variance for a single-phase estimation in an interferometer with two arms, both with transmissivity $\eta$, is $[(1-\eta) / \eta](1 / N)$. Thus, the asymptotic total variance for the separate estimation of $\mathfrak{p}$ phases with $N$ total photons is $\sum_{x=1}^{\mathfrak{p}}[(1-\eta) / \eta](1 / n)=[(1-\eta) / \eta]\left(\mathfrak{p}^{2} / N\right)$, where $n=$ $(N / \mathfrak{p})$ photons are allocated to each phase. This means that our multiparameter bound imposes a strong limitation: Even the most general strategy allowed by quantum mechanics can give at most a factor 4 improvement in the asymptotic total variance.

This potential advantage granted by simultaneous estimation comes from not needing separate reference beams for measuring each phase. Indeed, a simple calculation shows that if we assume to have a free external phase 
reference [92], the single-phase variance obtained with a squeezed coherent state is optimal, yielding [ $1-$ $\eta) / 4 \eta](1 / \bar{n})$ in the limit of a large average number of photons $\bar{n}$; see also Ref. [94]. The bound (32) is therefore equivalent to having a free phase reference and estimating each phase with a mean photon number $\bar{n}=N / \mathfrak{p}$. This observation is similar to the one made in Ref. [90], where the advantage of simultaneous over separate strategies was studied in idealized lossless models where asymptotic Heisenberg scaling is achievable. Furthermore, in the lossless scenario restricted to classical light (thus, with SQL precision), simultaneous estimation gives again a factor 4 advantage for $\mathfrak{p} \gg 1$, as shown by explicitly finding the optimal probe state [87]. While practical schemes to attain the bound (32) asymptotically (both in $N$ and $\mathfrak{p}$ ) are currently not known for the lossy case, the physical motivation of the advantage we discussed suggests that the bound should be attainable, similar to the lossless case.

\section{Multiple losses}

Estimating optical losses is another paradigmatic task where quantum light offers an advantage over classical light, even if not in terms of scaling with the total number of photons. In real-life applications, multiple losses must be estimated simultaneously, e.g., for the imaging of absorbing samples or in absorption spectroscopy. This problem has been studied in Ref. [95] where it was proven that simultaneous estimation offers no advantage over estimating each parameter individually. However, similar to the situation discussed in Sec. IV B for multiple phases, previous results are based on a mode description (thus, a parallel strategy) and, citing Ref. [95], "it remains to be seen if sequential adaptive estimation strategies can yield still more quantum enhancement." Using our tools, we answer this question and show that sequential adaptive schemes do not yield any additional enhancement.

In a particle description we can model multiple losses with the following Kraus operators:

$K_{0}=\left[\begin{array}{c}\operatorname{diag}\left(\sqrt{\eta_{1}}, \ldots, \sqrt{\eta_{\mathfrak{p}}}\right) \\ 0, \ldots, 0\end{array}\right] \quad K_{i}=\sqrt{1-\eta_{i}}|\mathfrak{p}+1\rangle\langle i|$.

Since each loss can be thought of as a beam splitter, as shown in Fig. 3(c), it is natural and useful to parametrize the problem in terms of the mixing angles $\boldsymbol{\phi}$ instead of the transmissivities $\boldsymbol{\eta}$, these parameters are related as $\cos \left(\phi_{j}\right)=\sqrt{\eta_{j}}$. With this parametrization and these Kraus operators, we obtain the bounds $\mathfrak{B}=\mathfrak{F}=4$ for $h_{x}=0 \forall x$, which correspond to $\operatorname{Tr} \mathcal{F} \leq 4 N$ for $N$ uses of the single-photon channel. This upper bound on the trace is saturated by the optimal QFI matrix obtained in Ref. [95] from a mode description: $4 \operatorname{diag}\left(\bar{n}_{1}, \ldots, \bar{n}_{\mathfrak{p}}\right)$ where $\bar{n}_{x}$ is the average number of photons used to probe each parameter $\phi_{x}$ (for probe states without a fixed photon number, the SQL bound applies to each sector so that $N$ can safely be substituted by the average photon number [6]). Since there is no measurement incompatibility, the QFI matrix gives in turn an attainable Cramér-Rao bound, and when photons are evenly distributed as $\bar{n}_{x}=(N / \mathfrak{p}) \forall x$, all inequalities in Eq. (6) are tight.

\section{Phase and loss}

Here we consider the problem of simultaneously estimating the phase shift and the loss (absorption) induced by a sample in one arm of a two-arm interferometer (the case of symmetric loss in both arms is simpler and fully solved in Ref. [29]). This problem is a paradigmatic example of a trade-off between the errors on the two parameters [57]. On the one hand, this problem shows measurement incompatibility, making an analysis in terms of the QFI not complete; for particular probe states and low photon numbers, the fundamental Holevo Cramér-Rao bound is studied in Refs. [40,45]. On the other hand, the problem shows also probe incompatibility: Single-mode Fock states are optimal for loss estimation [96] but phase insensitive.

Again, we model this metrological problem using a particle description of photons so that we can describe a single photon as a qubit [91]. The channel experienced by a single photon is thus described by the following Kraus operators:

$K_{0}=\left[\begin{array}{cc}\sqrt{\eta} e^{-i \theta} & 0 \\ 0 & 1 \\ 0 & 0\end{array}\right], \quad K_{1}=\left[\begin{array}{cc}0 & 0 \\ 0 & 0 \\ \sqrt{1-\eta} & 0\end{array}\right]$,

where the extra output dimension accounts for lost photons, and we want to estimate the phase $\theta$ and the transmissivity $\eta$.

As intuitively expected, with a single use of the channel there is indeed probe incompatibility and we obtain

$$
\mathfrak{I}=2\left(\frac{1-\eta}{\eta+\sqrt{\eta}-\sqrt{2(1+\sqrt{\eta})}}\right)^{2},
$$

a strictly increasing function of $\eta$ in the range $0 \leq \eta \leq 1$ that spans the values $1 \leq \mathfrak{I} \leq \frac{32}{25}$. Alternatively, probe incompatibility can be observed from the fact that the total channel QFI with $q_{i}=1$ is equal to the optimal QFI about the transmissivity $\mathfrak{F}=\mathfrak{F}_{\eta}=\{1 /[\eta(1-\eta)]\}$.

On the contrary, the asymptotic bound indicates that there is no incompatibility as we have $\mathfrak{J}_{\infty}=1$ and $\mathfrak{B}=\mathfrak{B}_{\theta}+\mathfrak{B}_{\eta}$. Further evidence of a reduction of probe incompatibility as $N$ increases is obtained by a numerical evaluation of the single-use bound for $\mathrm{N}$-photon channels. More details on these calculations are found in Appendix H 3. 


\section{E. Phase and dephasing}

Simultaneous estimation of an optical phase and its phase-diffusion coefficient is another well-known twoparameter problem $[56,97,98]$. At the single-photon level, the evolution is described by the following Kraus operators:

$$
K_{0}=\sqrt{\frac{1+\eta}{2}}\left[\begin{array}{cc}
e^{i \theta} & 0 \\
0 & 1
\end{array}\right], \quad K_{1}=\sqrt{\frac{1-\eta}{2}}\left[\begin{array}{cc}
e^{i \theta} & 0 \\
0 & -1
\end{array}\right] .
$$

It is known that there is no probe incompatibility for $N=1$, while it reappears for $N \geq 2$ and then again decreases with $N$ [29] and vanishes asymptotically [97]. Our bounds (20) and (12) show that both with a single use of the channel and asymptotically there is no probe incompatibility: $\mathfrak{I}=\mathfrak{J}_{\infty}=1$. Details on this calculation are presented in Appendix H 4. This asymptotic disappearance of incompatibility agrees with the results of a direct QFI maximization in parallel strategies reported in Refs. [29,97].

\section{APPLICATION TO QUANTUM-CHANNEL DISCRIMINATION}

In this section, we draw a connection between bounds on the total QFI introduced in Sec. III and the problem of discriminating between several channels. First, we generalize the framework to arbitrary quantum-channel discrimination tasks. This approach is particularly suited to those problems where there is some reference channel to which the channels are naturally related. In this framework, we derive an inequality that we call a speed limit for the discrimination of multiple noisy channels. As an application, we derive bounds on the performance of a timecontinuous version of Grover's algorithm in the presence of noise, revisiting the approach of Ref. [61]. Our new bounds allow us to close a gap in the proof that was left open as a conjecture.

\section{A. Background notions}

\section{Probability of error}

The error in discriminating among $\mathfrak{p}$ states $\rho_{n}$ with prior probability $p_{x}$ is given by [99]

$$
P_{H}\left(\left\{\rho_{x}, p_{x}\right\}\right):=1-\max _{\sum_{x} \Pi_{x}=\mathbb{1}} \sum_{x=1}^{\mathfrak{p}} p_{x} \operatorname{Tr}\left(\rho_{x} \Pi_{x}\right),
$$

where $H$ stands for the "Helstrom error" and $\left\{\Pi_{x}\right\}$ is a $\mathfrak{p}$-outcome POVM. For $\mathfrak{p}=2$ and equal priors $p_{1}=$ $p_{2}=1 / 2$, it reduces to

$$
P_{H}\left(\rho_{1}, \rho_{2}\right)=\frac{1}{2}\left[1-D_{\text {tr }}\left(\rho_{1}, \rho_{2}\right)\right],
$$

where

$$
D_{\text {tr }}\left(\rho_{1}, \rho_{2}\right):=\frac{1}{2}\left\|\rho_{1}-\rho_{2}\right\|_{1}
$$

is the trace distance, and $\|A\|_{1}=\operatorname{Tr} \sqrt{A^{\dagger} A}$ is the trace norm, i.e., the sum of the singular values of $A$. It is useful to introduce the Fuchs-van de Graaf inequalities between the trace distance and the fidelity [100]

$$
1-F\left(\rho_{1}, \rho_{2}\right) \leq D_{\text {tr }}\left(\rho_{1}, \rho_{2}\right) \leq \sqrt{1-F\left(\rho_{1}, \rho_{2}\right)^{2}},
$$

where we define the fidelity as

$$
F\left(\rho_{1}, \rho_{2}\right):=\left\|\sqrt{\rho_{1}} \sqrt{\rho_{2}}\right\|_{1}=\operatorname{Tr} \sqrt{\sqrt{\rho_{1}} \rho_{2} \sqrt{\rho_{1}}} .
$$

There is no closed-form solution for the generic multihypothesis problem, but it is possible to find upper and lower bounds. A lower bound in terms of binary discrimination is the following [101]:

$$
P_{H}\left(\left\{\rho_{x}, p_{x}\right\}\right) \geq \frac{1}{2}\left(1-\frac{1}{\mathfrak{p}-1} \sum_{1 \leq x<y \leq \mathfrak{p}}\left\|p_{x} \rho_{x}-p_{y} \rho_{y}\right\|_{1}\right) .
$$

\section{Bures angle}

The angular Bures distance or Bures angle is defined in terms of the fidelity [102]

$$
D_{A}\left(\rho_{1}, \rho_{2}\right):=\arccos F\left(\rho_{1}, \rho_{2}\right),
$$

and in particular, the infinitesimal version is related to the QFI

$$
D_{A}\left(\rho_{\theta}, \rho_{\theta+d \theta}\right)=\frac{1}{2} \sqrt{\mathcal{F}\left(\rho_{\theta}\right)} d \theta
$$

where we introduce a suitable smooth parametrization. Crucially, $D_{A}\left(\rho_{1}, \rho_{2}\right)$ is the length of a geodesic path between $\rho_{1}$ and $\rho_{2}$ with respect to this infinitesimal metric $[103,104]$; thus, for any smooth parametrization such that $\rho_{1}=\rho_{\theta=0}$ and $\rho_{2}=\rho_{\theta=\theta^{*}}$, we have

$$
D_{A}\left(\rho_{1}, \rho_{2}\right) \leq \frac{1}{2} \int_{0}^{\theta^{*}} \sqrt{\mathcal{F}\left(\rho_{\theta}\right)} d \theta .
$$

We use the second inequality (40) to upper bound the trace distance with the Bures angle

$D_{\text {tr }}\left(\rho_{1}, \rho_{2}\right) \leq \sqrt{1-\cos ^{2}\left[D_{A}\left(\rho_{1}, \rho_{2}\right)\right]} \leq D_{A}\left(\rho_{1}, \rho_{2}\right)$, 


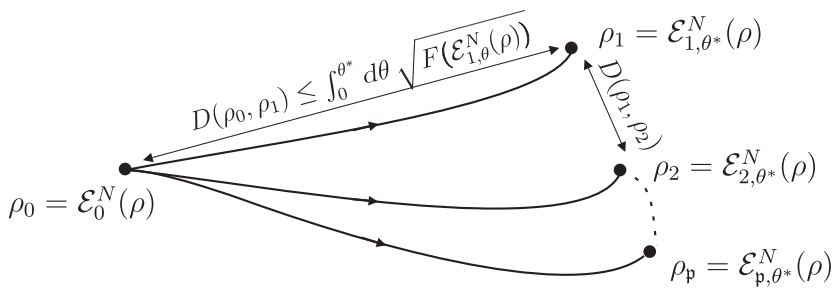

FIG. 4. Intuitive representation of the geometrical argument connecting the error in the discrimination of $\mathfrak{p}$ quantum channels with the precision of random sensing.

where we use the inequality $\cos ^{2} \xi \geq 1-\xi^{2}$ for $0 \leq \xi \leq \pi / 2$.

\section{B. Reference-state bound for state discrimination}

Thanks to inequality (46) and the triangle inequality, the trace distance can be upper bounded as

$$
D_{\text {tr }}\left(\rho_{x}, \rho_{y}\right) \leq D_{A}\left(\rho_{x}, \rho_{0}\right)+D_{A}\left(\rho_{y}, \rho_{0}\right),
$$

where we introduce an arbitrary reference state $\rho_{0}$. Now we make the crucial assumption that the states $\rho_{x}$ and $\rho_{y}$ are parametrized by a parameter $\theta$ such that $\rho_{x}=\rho_{x, \theta=\theta^{*}}$, $\rho_{y}=\rho_{y, \theta=\theta^{*}}$, and $\rho_{0}=\rho_{x, \theta=0}=\rho_{y, \theta=0}$, and thanks to Eq. (45), we have

$D_{\text {tr }}\left(\rho_{x}, \rho_{y}\right) \leq \frac{1}{2}\left[\int_{0}^{\theta^{*}} d \theta \sqrt{\mathcal{F}\left(\rho_{x, \theta}\right)}+\sqrt{\mathcal{F}\left(\rho_{y, \theta}\right)}\right]$.

The intuition of using the geodesic and the triangle inequalities is pictorially represented in Fig. 4.

Now we focus on discriminating $\mathfrak{p}$ states with uniform prior $q_{x}=(1 / \mathfrak{p})$ and apply Eq. (42) to find a lower bound on the error from pairwise discrimination. Thanks to Eq. (48), the sum appearing in Eq. (42) is upper bounded as follows:

$$
\begin{aligned}
\sum_{1 \leq x<y \leq \mathfrak{p}} D_{\operatorname{tr}}\left(\rho_{x}, \rho_{y}\right) & \leq \frac{(\mathfrak{p}-1)}{2} \int_{0}^{\theta^{*}} d \theta \sum_{x=1}^{\mathfrak{p}} \sqrt{\mathcal{F}\left(\rho_{x, \theta}\right)} \\
& \leq \frac{(\mathfrak{p}-1) \sqrt{\mathfrak{p}}}{2} \int_{0}^{\theta^{*}} d \theta \sqrt{\sum_{x=1}^{\mathfrak{p}} \mathcal{F}\left(\rho_{x, \theta}\right)},
\end{aligned}
$$

where first we use the identity $\sum_{1 \leq x<y \leq p}\left(q_{x}+q_{y}\right)=$ $(\mathfrak{p}-1) \sum_{x} q_{x}$ and then the Jensen inequality $\sum_{i=1}^{\mathfrak{p}} \sqrt{q_{i}} \leq$ $\sqrt{\mathfrak{p}} \sqrt{\sum_{i=1}^{\mathfrak{p}} q_{i}}$. Using Eq. (49) in conjunction with Eq. (42), we obtain

$$
\int_{0}^{\theta^{*}} d \theta \sqrt{\frac{1}{\mathfrak{p}} \sum_{x=1}^{\mathfrak{p}} \mathcal{F}\left(\rho_{x, \theta}\right)} \geq 1-2 P_{H}\left(\left\{\rho_{x}, \mathfrak{p}^{-1}\right\}\right) .
$$

\section{Speed limits for discriminating noisy quantum channels}

The derivations of the previous section have limited utility for discriminating an ensemble of quantum states. In general, for a fixed set of states the sum $\sum_{x=1}^{\mathfrak{p}} \mathcal{F}\left(\rho_{x}\right)=$ $O(\mathfrak{p})$, since there are no issues with probe incompatibility. Thanks to the additivity of the QFI, we thus get a trivial bound (constant in the number $\mathfrak{p}$ of states) on the number of copies $N$ needed to discriminate with fixed accuracy $N \geq O(1)$. This is a weak and noninformative bound, since it is known that the dependence on $\mathfrak{p}$ should be logarithmic [105]. However, such bounds reveal their usefulness when applied to channel discrimination, where we can take advantage of general and powerful upper bounds on the total QFI that we introduced. In particular, we focus on the case when the HKS conditions are satisfied for all channels, which is always true for fullrank channels.

We consider the discrimination of an ensemble of channels $\left\{\mathcal{E}_{x}\right\}_{x=1}^{\mathfrak{p}}$ with uniform prior, assuming $N$ repeated uses and a general adaptive strategy with auxiliary systems. We fix a target error $\varepsilon$, and we find a lower bound on the number $N$ of uses of the channel (i.e., channel queries) needed to go below the error threshold. The problem amounts to identification of the minimal value of $N$ for which

$$
\min _{\rho_{0},\left\{\mathcal{V}_{i}\right\}} P_{H}\left(\left\{\mathcal{E}_{x}^{N}\left(\rho_{0}\right), \mathfrak{p}^{-1}\right\}\right) \leq \varepsilon,
$$

where $\mathcal{E}_{x}^{N}\left(\rho_{0}\right)$ is the overall channel of the adaptive strategy, as defined in Sec. III.

Instead of a reference state, we now introduce a reference channel $\mathcal{E}_{0}$ and a suitable parametrization such that $\mathcal{E}^{x}=$ $\mathcal{E}_{\theta=\theta^{*}}^{x}$ and $\mathcal{E}_{0}=\mathcal{E}_{x, \theta=0}$, as depicted in Fig. 4. In this way, we can apply Eq. (50) and obtain

$$
\int_{0}^{\theta^{*}} d \theta \sqrt{\frac{1}{\mathfrak{p}} \min _{\rho_{0},\left\{\mathcal{V}_{i}\right\}} \sum_{x=1}^{\mathfrak{p}} \mathcal{F}\left(\mathcal{E}_{x, \theta}^{N}\left(\rho_{0}\right)\right)} \geq 1-2 \varepsilon .
$$

Here we see that the bounds on the total QFI $\mathfrak{F}^{N}$ that we derive in Sec. III are useful even when considering different channels. In particular, if there exists a parametrization such that the HKS conditions are satisfied for all channels, we obtain the following inequality:

$$
N \geq \frac{\mathfrak{p}(1-2 \varepsilon)^{2}}{\left(\int_{0}^{\theta^{*}} d \theta \sqrt{\mathfrak{B}(\theta)}\right)^{2}},
$$


where we highlight that the SQL bound $\mathfrak{B}(\theta)$ generally depends on the value $\theta$ at which it is evaluated. We call this inequality a speed limit, a term which is more appropriate when taking a continuous limit, as done in Appendix D for Markovian noise.

We highlight that the speed limit (53) gives a nontrivial result in the regime where $\varepsilon$ is small, i.e., in the regime of large distinguishability. On the contrary, for $\varepsilon=\frac{1}{2}$ the bound is completely trivial, yielding $N \geq 0$. We also remark that in general it is not possible to perfectly discriminate between noisy quantum channels with a finite number of uses [106] (or finite time for Markovian noise [107]), unlike for unitary channels [108].

If instead of fixing a target error (51) we require an identical target fidelity between all pairs of final states, which in terms of the Bures angle means

$$
D_{A}\left[\mathcal{E}_{x}^{N}\left(\rho_{0}\right), \mathcal{E}_{y}^{N}\left(\rho_{0}\right)\right]=\delta, \quad \forall x \neq y,
$$

we can derive a slightly tighter bound similar to Ref. [61]

$$
N \geq \frac{\mathfrak{p} \delta^{2}}{\left(\int_{0}^{\theta^{*}} d \theta \sqrt{\mathfrak{B}(\theta)}\right)^{2}}
$$

\section{Loss of computational speed-up in the presence of noise}

Following Ref. [61], we consider the time-continuous version of Grover's algorithm proposed by Farhi and Gutmann [109]. The problem of finding a marked element $x$ of a database of size $d$ is recast as determining which oracle Hamiltonian $G_{x}=|x\rangle\langle x|$ generates the evolution $U_{\omega, \tau}^{x}=e^{-i \omega \tau G_{x}}$, where $\tau$ and $\omega$ are assumed to be known. When the oracle is noiseless, it is possible to find the marked element with a total runtime $O(\sqrt{d})$ instead of the classical $O(d)$ by appropriately driving the system.

It has been shown that a noisy oracle usually destroys the quantum advantage, although this behavior was shown only for specific noise models $[110,111]$. We study the same problem, and we confirm the same loss of advantage for a couple of new noise models, highlighting the generality of the methodology employed.

In the following, we focus on noisy channels that commute with the unitary evolution so that we can choose $\mathcal{E}_{x, \omega, \tau}=\mathcal{E}_{\tau} \circ \mathcal{U}_{x, \omega, \tau}$ where $\mathcal{U}(\cdot)$ denotes the map $U \cdot U^{\dagger}$. Moreover we focus on Markovian noise with a semigroup property $\mathcal{E}_{\tau+\tau^{\prime}}=\mathcal{E}_{\tau} \circ \mathcal{E}_{\tau^{\prime}}$. Using the speed limit that we introduced, we obtain a bound on the total run-time $T$ under the most general entanglement-enhanced adaptive strategy. Thanks to the Markovianity assumption, the strongest bound is obtained in the limit $\tau \rightarrow 0$, i.e., when the control operations are applied as frequently as possible. In this case, when invoking the metrological bound, we are in fact using a direct generalization of the derived bounds adapted to the estimation of multiple Hamiltonian parameters with arbitrary Markovian noise under the most general adaptive strategy, following the same approach used in Refs. [4,5,75]; see Appendix D for details.

\section{Uniform qudit dephasing}

We consider the following uniform dephasing channel:

$$
\mathcal{E}(\rho)=\eta \rho+(1-\eta) \Delta(\rho),
$$

where $\Delta(\rho)$ is the completely dephasing channel with respect to the basis of the oracle Hamiltonians, i.e., $\Delta(\rho)=\operatorname{diag}(\rho)$. We consider the total QFI of the ensemble $\left\{\mathcal{E} \mathcal{U}_{x, \theta}\right\}_{x=1}^{d}$, where the unitaries $U_{x, \theta}=e^{-i \theta G_{x}}$. The corresponding SQL bound (20) can be evaluated analytically

$$
\mathfrak{B}_{\theta}(\eta)=\frac{4 \eta}{1-\eta} \frac{d-1}{d+\frac{2}{\eta}} \underset{[d \rightarrow \infty]}{\rightarrow} \frac{4 \eta}{1-\eta} ;
$$

the detailed calculation is relegated to Appendix H 5.

To apply the above result to the problem of identifying the oracle Hamiltonian, we change the parametrization to $\omega=\theta / \tau$, and we choose $\eta_{\gamma, \tau}=e^{-\gamma \tau}$ to obtain the Markovian dephasing channel. For a total run-time $T=N \tau$, the best bound for frequency estimation is thus,

$$
\mathfrak{B}_{\omega}=\lim _{\tau \rightarrow 0} \tau \mathfrak{B}\left(\eta_{\gamma, \tau}\right)=\frac{4(d-1)}{\gamma(d+2)} \leq \frac{4}{\gamma} .
$$

The bound $\mathfrak{B}_{\omega}$ can alternatively be obtained directly from the Lindblad operators $\left\{L_{i}=\sqrt{\gamma}|i\rangle\langle i|\right\}_{i=1}^{d}$ using the approach presented in Appendix D. Using inequality (58) together with Eq. (55), we obtain a lower bound on the total run-time $T$ required to reach the desired Bures angle $\delta$ between all possible final states:

$$
T \geq d \frac{\gamma \delta^{2}}{4 \omega^{2}}
$$

In particular, a successful identification of all possible generators $G_{x}$ means that all final states must be perfectly distinguishable, thus, $\delta=(\pi / 2)$. The corresponding bound on the run-time is then $T \geq d\left[\left(\gamma \pi^{2}\right) / 16 \omega^{2}\right]$. This closes the conjecture of Ref. [61].

\section{Erasure noise}

We notice that the SQL bound (30) for diagonal generators can be applied to our problem of discriminating the oracle Hamiltonians in the presence of Markovian erasure noise. We just need to substitute $\eta_{\gamma, \tau}=e^{-\gamma \tau}$ as the noise parameter to obtain 


$$
\mathfrak{B}_{\omega}=\lim _{\tau \rightarrow 0} \tau \mathfrak{B}_{\theta}\left(\eta_{\gamma, \tau}\right)=\frac{4(d-1)}{d \gamma} \leq \frac{4}{\gamma}
$$

Finally, from this we get the same bound on the runtime (59) as for qudit dephasing.

\section{DISCUSSION AND CONCLUSIONS}

As multiparameter quantum metrology moves to practical applications it becomes crucial to understand its advantages and limitations. Our first contribution in this regard is conceptual: We define a new measure of metrological incompatibility that depends only on the local geometry of the parametric family of quantum channels. In this paper, we evaluate lower bounds on this measure by choosing a particular parametrization and considering only probe incompatibility, which indeed represents the main challenge. However, further efforts to evaluate this incompatibility measure are certainly needed, since it is a quantity that captures all obstructions imposed both by quantum mechanics and classical statistics on the task of accurately learning multiple properties of a single quantum channel at once. From a different point of view, quantum incompatibility is understood as the impossibility to jointly implement two or more input-output devices as components of a larger device [112], leading to a notion of incompatible quantum channels $[113,114]$. Understanding possible connections between these diverse notions of incompatibility is a challenging and interesting open question.

As our main technical result, we derive widely applicable and computable multiparameter metrological bounds that apply to several different scenarios. On the one hand, this is immediately useful to impose strong limitations on the best achievable precision and to assess the optimality of feasible protocols. Indeed, in Sec. IV we show that the advantage of simultaneous multiphase estimation is at most a factor 4 in the total variance, and that existing schemes for estimating multiple losses cannot be improved upon using adaptive strategies. On the other hand, taking into account probe incompatibility is a fundamental first step toward a full asymptotic theory of noisy multiparameter quantum metrology. However, to successfully extend the single-parameter analysis [8], it is crucial to investigate the attainability of the bounds and to devise optimal asymptotic strategies. This is the next step needed to uncover the full potential of multiparameter quantum metrology in realistic scenarios.

Another important extension toward realistic situations is to go beyond models where noise acts independently. In this regard, the optimal precision for single-parameter metrology with non-Markovian noise was studied using the seminal purification argument of Ref. [60] that we also use, obtaining a way to numerically evaluate it with an SDP [115]. While work on this topic is overall still in the early stages, we foresee that our extension to multiple parameters will be fundamental in this scenario as well.
Speaking of realistic precision bounds, a further word of caution is in order. When Heisenberg scaling is possible, a careful analysis of truly optimal protocols generally does not agree with a naive local approach (see, e.g., Refs. [116-118]), such as the one we apply in this paper. This discrepancy becomes even more critical for multiparameter problems, where it might affect the scaling of the precision with the number of parameters [90]. Luckily, in the noisy SQL scenario there is no such problem [117], and therefore, the analysis we present in this paper holds even for truly optimal protocols.

On a more practical level, in the examples we consider our bounds show that the addition of noise on top of a unitary parameter encoding, while prohibiting Heisenberg scaling, does not affect the amount of probe incompatibility. This also means that the original advantage (or lack thereof) of simultaneous estimation strategies over separable ones appears to be preserved. This is certainly an intriguing observation that ought to be investigated more in depth in the future. In particular, proving a practical advantage of simultaneous strategies even under inevitably noisy working conditions could have important consequences for future technological applications.

Finally, as we show by studying the problem of a noisy Grover search, the applicability of these theoretical tools extends even beyond quantum metrology. Indeed, tools from quantum estimation theory are now routinely used in quantum thermodynamics [119,120], quantum speed limits [121], quantum algorithms, and quantum machine learning [122]. In particular, single-parameter fundamental metrological bounds have recently found an application in the theory of quantum-error correction [123-125], providing new perspectives and results. Accordingly, we expect that such a fertile interplay of different research fields will become even more relevant in the future. We hope that our results will play a role in these endeavors.

\section{ACKNOWLEDGMENTS}

We thank Wojtek Górecki for fruitful discussions. We also thank Janek Kołodyński and Christian Gogolin for sharing with us some of their notes and thoughts on proving the conjecture in Ref. [61]. This work is supported by the National Science Center (Poland) Grant No. 2016/22/E/ ST2/00559.

\section{APPENDIX A: DERIVATION OF CHANNEL INCOMPATIBILITY BOUND (9)}

The incompatibility measure (8) can be lower bounded using the QCRB

$$
\mathfrak{J}^{*}\left(\mathcal{E}_{\boldsymbol{\theta}}\right) \geq \max _{\left\{\mathbf{w}_{x}\right\}}\left(\frac{\min _{\rho} \operatorname{tr}\left[W \mathcal{F}^{-1}\left(\mathcal{E}_{\boldsymbol{\theta}}(\rho)\right)\right]}{\sum_{x} \mathbf{w}_{x}^{T} \mathcal{F}^{-1}\left(\mathcal{E}_{\boldsymbol{\theta}}\left(\rho_{x}^{*}\right)\right) \mathbf{w}_{x}}\right),
$$

where we denote by $\rho_{x}^{*}=\operatorname{argmin}_{\rho_{x}} \mathbf{w}_{x}^{T} \mathcal{F}^{-1}\left(\mathcal{E}_{\boldsymbol{\theta}}\left(\rho_{x}\right)\right) \mathbf{w}_{x}$ the optimal input probe for estimating the $x$ th parameter ( $x$ th scalar function). One could also obtain a tighter lower 
bound by using the Holevo Cramér-Rao bound [31,34] on the numerator of Eq. (8) to retain the (asymptotic) effect of measurement incompatibility.

By rescaling the cost vectors

$$
\mathbf{w}_{x} \rightarrow \frac{\mathbf{w}_{x}}{\sqrt{\mathbf{w}_{x}^{T} \mathcal{F}^{-1}\left(\mathcal{E}\left(\rho_{x}^{*}\right)\right) \mathbf{w}_{x}}},
$$

we recast the rhs of Eq. (A1) as

$$
\mathfrak{J}^{*}\left(\mathcal{E}_{\boldsymbol{\theta}}\right) \geq \frac{1}{\mathfrak{p}} \max _{\left\{\mathbf{w}_{x}\right\}} \min _{\rho} \sum_{x} \frac{\mathbf{w}_{x}^{T} \mathcal{F}^{-1}\left(\mathcal{E}_{\boldsymbol{\theta}}(\rho)\right) \mathbf{w}_{x}}{\mathbf{w}_{x}^{T} \mathcal{F}^{-1}\left(\mathcal{E}_{\boldsymbol{\theta}}\left(\rho_{x}^{*}\right)\right) \mathbf{w}_{x}} .
$$

We now lower bound this quantity further by restricting the class of cost vectors $\mathbf{w}_{x}$ to belong to the set of eigenvectors of $\mathcal{F}\left(\mathcal{E}\left(\rho_{x}^{*}\right)\right)$ :

$$
\mathfrak{J}^{*}\left(\mathcal{E}_{\boldsymbol{\theta}}\right) \geq \frac{1}{\mathfrak{p}} \max _{\left\{\mathbf{w}_{x} \in \mathcal{W}_{x}\right\}} \min _{\rho} \sum_{x} \frac{\mathbf{w}_{x}^{T} \mathcal{F}^{-1}\left(\mathcal{E}_{\boldsymbol{\theta}}(\rho)\right) \mathbf{w}_{x}}{\mathbf{w}_{x}^{T} \mathcal{F}^{-1}\left(\mathcal{E}_{\boldsymbol{\theta}}\left(\rho_{x}^{*}\right)\right) \mathbf{w}_{x}},
$$

where by $\mathcal{W}_{x}$ we denote the set of eigenvectors of $\mathcal{F}\left(\mathcal{E}\left(\rho_{x}^{*}\right)\right)$. The restriction to eigenvectors of $\mathcal{F}\left(\mathcal{E}\left(\rho_{x}^{*}\right)\right)$ is a natural assumption, since when minimizing the cost for a given single parameter the optimal state will tend to carry as much information about the desired parameter with as little correlations as possible with the others (as potential correlations increase the diagonal entries of the inverse QFI matrix). Moreover, if the minimization of the cost for a given parameter $x$, leads to a QFI matrix with nondiagonal entries (nonvanishing correlations), this indicates that the choice of parametrization is not natural for the model considered. We now use the eigenvector property to take the vectors under the inverse operation and get

$\mathfrak{J}^{*}\left(\mathcal{E}_{\boldsymbol{\theta}}\right) \geq \frac{1}{\mathfrak{p}} \max _{\left\{\mathbf{w}_{x} \in \mathcal{W}_{x}\right\}} \min _{\rho} \sum_{x} \frac{\mathbf{w}_{x}^{T} \mathcal{F}^{-1}\left(\mathcal{E}_{\boldsymbol{\theta}}(\rho)\right) \mathbf{w}_{x}}{\left[\mathbf{w}_{x}^{T} \mathcal{F}\left(\mathcal{E}_{\boldsymbol{\theta}}\left(\rho_{x}^{*}\right)\right) \mathbf{w}_{x}\right]^{-1}\left(\mathbf{w}_{x}^{T} \mathbf{w}_{x}\right)^{2}}$.

We can now apply a series of inequalities analogous to Eq. (6), and arrive at

$$
\begin{aligned}
& \mathfrak{J}^{*}\left(\mathcal{E}_{\boldsymbol{\theta}}\right) \\
& \geq \frac{1}{\mathfrak{p}} \max _{\left\{\mathbf{w}_{x} \in \mathcal{W}_{x}\right\}} \min _{\rho} \sum_{x} \frac{\left[\mathbf{w}_{x}^{T} \mathcal{F}\left(\mathcal{E}_{\boldsymbol{\theta}}(\rho)\right) \mathbf{w}_{x}\right]^{-1}}{\left[\mathbf{w}_{x}^{T} \mathcal{F}\left(\mathcal{E}_{\boldsymbol{\theta}}\left(\rho_{x}^{*}\right)\right) \mathbf{w}_{x}\right]^{-1}} \\
& \geq \mathfrak{p} \max _{\left\{\mathbf{w}_{x} \in \mathcal{W}_{x}\right\}} \min _{\rho} \frac{1}{\sum_{x}\left[\mathbf{w}_{x}^{T} \mathcal{F}\left(\mathcal{E}_{\boldsymbol{\theta}}\left(\rho_{x}^{*}\right)\right) \mathbf{w}_{x}\right]^{-1}\left[\mathbf{w}_{x}^{T} \mathcal{F}\left(\mathcal{E}_{\boldsymbol{\theta}}(\rho)\right) \mathbf{w}_{x}\right]} .
\end{aligned}
$$

Finally, when we fix $\mathbf{w}_{x}$, i.e., choose a particular parametrization, we arrive at Eq. (9).

\section{APPENDIX B: BOUND FOR THE ADAPTIVE STRATEGY: PROOF OF THEOREM 2}

To derive the bound, we need the following inequality for the operator norm.
Lemma B.1: Given a set of $\mathfrak{p}$ square matrices $\left\{A^{x}\right\}_{x=1}^{\mathfrak{p}}$ and $\mathfrak{p}$ sets of rectangular matrices $\left\{L_{k_{x}}^{x}\right\}_{k_{x}=1}^{n_{x}}$ (with compatible dimensions), we have the following inequality:

$$
\left\|\sum_{x=1}^{\mathfrak{p}} \sum_{k_{x}=1}^{n_{x}} L_{k_{x}}^{x^{\dagger}} A^{x} L_{k_{x}}^{x}\right\| \leq \max _{x}\left\{\left\|A^{x}\right\|\right\}\left\|\sum_{x=1}^{\mathfrak{p}} \sum_{k_{x}=1}^{n_{x}} L_{k_{x}}^{x^{\dagger}} L_{k_{x}}^{x}\right\| .
$$

Proof--Let us define two matrices

$$
\begin{gathered}
\tilde{L}^{\dagger}=\left[L_{1}^{1 \dagger}, \ldots, L_{n_{1}}^{1 \dagger}, L_{1}^{2 \dagger}, \ldots, L_{n_{2}}^{2 \dagger}, \ldots, L_{1}^{p^{\dagger}}, \ldots, L_{n_{p}}^{p^{\dagger}}\right], \\
\tilde{A}=\bigoplus_{i=1}^{\mathfrak{p}} \mathbb{1}_{n_{i}} \otimes A_{i} ;
\end{gathered}
$$

i.e., $\tilde{A}$ is a block diagonal matrix with each $A_{i}$ repeated in $n_{i}$ consecutive diagonal blocks. We have that

$$
\tilde{L}^{\dagger} \tilde{A} \tilde{L}=\sum_{x=1}^{\mathfrak{p}} \sum_{j=1}^{n_{x}} L_{j}^{x \dagger} A^{x} L_{j}^{x} .
$$

Thanks to the submultiplicativity of the operator norm, we get

$$
\left\|\sum_{x=1}^{\mathfrak{p}} \sum_{j=1}^{n_{x}} L_{j}^{x^{\dagger}} A^{x} L_{j}^{x}\right\|=\left\|\tilde{L} \tilde{L}^{\dagger} \tilde{A} \tilde{L}\right\| \leq\left\|\tilde{L}^{\dagger}\right\|\|\tilde{A}\|\|\tilde{L}\|,
$$

upon noticing that

$$
\begin{gathered}
\|\tilde{A}\|=\max _{x}\left\{\left\|A^{x}\right\|\right\}, \\
\left\|\tilde{L}^{\dagger}\right\|^{2}=\|\tilde{L}\|^{2}=\left\|\tilde{L}^{\dagger} \tilde{L}\right\|=\left\|\sum_{x=1}^{\mathfrak{p}} \sum_{j=1}^{n_{x}} L_{j}^{x^{\dagger}} L_{j}^{x}\right\|,
\end{gathered}
$$

we get the desired result (B1).

When reduced to the single-parameter case, we get

$$
\left\|\sum_{j=1}^{n} L_{k}^{\dagger} A L_{k}\right\| \leq\|A\|\left\|\sum_{j=1}^{n} L_{k}^{\dagger} L_{k}\right\| .
$$

With this technical tool, we can prove the main theorem.

Proof--First, we notice from the start that the final bound is expressed in terms of operator norms of the functions $\sum_{x} \alpha_{x}, \beta_{x}$, and $\sum_{x} \beta_{x}$, which depend only on the Kraus operator of a single copy of the channels $\mathcal{E}_{\theta_{x}}$. Crucially, these quantities are unchanged by additional unitaries applied between each use of the channel and by trivial extensions to auxiliary Hilbert spaces. Therefore, we derive the bound considering Kraus operators representing a direct $N$-fold sequential application of the channel: $\left(\mathcal{E}_{\theta_{x}}\right)^{N}$, but the results will be valid also in the general case described in the statement including control operations $\mathcal{V}_{i}$.

A natural choice of Kraus operators for $N$ sequential applications of the channels is $K_{\mathbf{k}_{x}}=K_{k_{N}}, \ldots, K_{k_{1}}$. This 
choice is not necessarily the optimal, but we use it to find an upper bound to the optimal channel bound (18) as follows:

$$
\begin{aligned}
& 4 \min _{K_{\mathbf{k}_{x}}}\left\|\sum_{x=1}^{\mathfrak{p}} q_{x} \sum_{\mathbf{k}_{x}} \partial_{\theta_{x}} K_{\mathbf{k}_{x}}^{\dagger} \partial_{\theta_{x}} K_{\mathbf{k}}\right\| \\
& =4 \min _{K_{\mathbf{k}_{x}}} \| \sum_{x} q_{x} \sum_{\mathbf{k}_{x}} \sum_{i, j=1}^{N} \\
& \quad \times K_{k_{1}}^{\dagger} \cdots \partial_{\theta_{x}} K_{k_{i}}^{\dagger} \cdots K_{k_{N}}^{\dagger} K_{k_{N}} \cdots \partial_{\theta_{x}} K_{k_{j}} \cdots K_{k_{1}} \| ;
\end{aligned}
$$

each multi-index $\mathbf{k}_{x}$ depends on $x$, yet for brevity we suppress the dependence on $x$ when expressing its components $k_{i}:=k_{x, i}$. For additional clarity in the final expressions, we reintroduce the dependence on $x$ when the components of the multi-indices disappear from the calculations. It is understood that the summation over $k_{i}$ is inside the summation over $x$ and the two cannot be exchanged, since the Kraus operators $K_{k_{x}}$ depend on $x$.

By splitting the sum over $i, j$ into diagonal and offdiagonal terms and using the triangle inequality and Eq. (B8), we get

$$
\mathfrak{F}_{\mathbf{q}}^{N} \leq 4 \min _{K_{\mathbf{k}_{x}}}\left\{\sum_{i=1}^{N}\left\|\sum_{x} q_{x} \sum_{k_{x}=1}^{r_{x}} \partial_{\theta_{x}} K_{k_{x}}^{\dagger} \partial_{\theta_{x}} K_{k_{x}}\right\|+\sum_{i<j}^{N} \| \sum_{x} q_{x} \sum_{k_{i} \cdots k_{j}} \partial_{\theta_{x}} K_{k_{i}}^{\dagger} \cdots K_{k_{j}}^{\dagger} \partial_{\theta_{x}} K_{k_{j}} \cdots K_{k_{i}}+\text { H.c. } \|\right\} .
$$

We introduce the anti-Hermitian operator

$$
i A_{i}^{x}=\sum_{k_{i+1} \cdots k_{j}} K_{k_{i+1}}^{\dagger} \cdots K_{k_{j}}^{\dagger} \partial_{\theta_{x}} K_{k_{j}} \cdots K_{k_{i+1}}
$$

so we can rewrite each term inside the second sum over $i<j$ in Eq. (B10) as follows:

$$
\| \sum_{x} q_{x} \sum_{k_{i} \cdots k_{j}} \partial_{\theta_{x}} K_{k_{i}}^{\dagger} \cdots K_{k_{j}}^{\dagger} \partial_{\theta_{x}} K_{k_{j}} \cdots K_{k_{i}}+\text { H.c. }\|=\| \sum_{x} q_{x} \sum_{k_{i}=1}^{r_{x}}\left(\sqrt{z_{x}} \partial_{\theta_{x}} K_{k_{i}}\right)^{\dagger} i A_{i}^{x}\left(\frac{K_{k_{i}}}{\sqrt{z_{x}}}\right)+\text { H.c. } \|,
$$

where we also introduce an additional set of real positive parameters $z_{x}>0$ that leaves the quantity unchanged. We can expand this quantity further

$$
\begin{aligned}
& \left\|\sum_{x} q_{x} \sum_{k_{x}=1}^{r_{x}}\left[\left(\sqrt{z_{x}} \partial_{\theta_{x}} K_{k_{x}}+i \frac{K_{k_{x}}}{\sqrt{z_{x}}}\right)^{\dagger} A_{i}^{x}\left(\sqrt{z_{x}} \partial_{\theta_{x}} K_{k_{x}}+i \frac{K_{k_{x}}}{\sqrt{z_{x}}}\right)-z_{x} \partial_{\theta_{x}} K_{k_{x}}^{\dagger} A_{i}^{x} \partial_{\theta_{x}} K_{k_{x}}-\frac{1}{z_{x}} K_{k_{x}}^{\dagger} A_{i}^{x} K_{k_{x}}\right]\right\| \\
& \leq \max _{x}\left(\left\|A_{i}^{x}\right\|\right)\left[\left\|\sum_{x} q_{x} \sum_{k_{x}=1}^{r_{x}}\left(\sqrt{z_{x}} \partial_{\theta_{x}} K_{k_{x}}+i \frac{K_{k_{x}}}{\sqrt{z_{x}}}\right)^{\dagger}\left(\sqrt{z_{x}} \partial_{\theta_{x}} K_{k_{x}}+i \frac{K_{k_{x}}}{\sqrt{z_{x}}}\right)\right\|+\left\|\sum_{x} z_{x} q_{x} \sum_{k} \partial_{\theta_{x}} K_{k_{x}}^{\dagger} \partial_{\theta_{x}} K_{k_{x}}\right\|+\left(\sum_{x} \frac{q_{x}}{z_{x}}\right)\right] \\
& \leq \max _{x}\left(\left\|A_{i}^{x}\right\|\right)\left[\left\|\sum_{x} q_{x} \sum_{k_{x}}^{r_{x}} \partial_{\theta_{x}} K_{k_{x}}^{\dagger} K_{k_{x}}\right\|+\left\|\sum_{x} z_{x} q_{x} \sum_{k_{x}} \partial_{\theta_{x}} K_{k_{x}}^{\dagger} \partial_{\theta_{x}} K_{k_{x}}\right\|+\left(\sum_{x} \frac{q_{x}}{z_{x}}\right)\right]
\end{aligned}
$$

where we have used the triangle inequality first, then inequality (B1) and then again the triangle inequality to obtain the last line. From Eq. (B8), we know that $\left\|A_{i}^{x}\right\| \leq\left\|\beta_{x}\right\|$, where $\beta_{x}=\sum_{k} \partial_{\theta_{x}} K_{k}^{\dagger} K_{k}=\partial_{\theta_{x}} \mathbf{K}^{\dagger} \mathbf{K}$; using this inequality, setting $z_{x}=z$, and performing the sums in Eq. (B10), we obtain

$$
\mathfrak{F}_{\mathbf{q}}^{N} \leq 4 \min _{\mathfrak{h}, z}\left\{N\left\|\sum_{x=1}^{\mathfrak{p}} q_{x} \alpha_{x}\right\|+N(N-1) \max _{x}\left(\left\|\beta_{x}\right\|\right)\left[\frac{1}{z}\left(\sum_{x=1}^{\mathfrak{p}} q_{x}\right)+z\left\|\sum_{x=1}^{\mathfrak{p}} q_{x} \alpha_{x}\right\|+\left\|\sum_{x=1}^{\mathfrak{p}} q_{x} \beta_{x}\right\|\right]\right\},
$$

and by performing the explicit minimization over the parameter $z$, we obtain the desired result Eq. (19).

We notice that it might be possible to obtain a tighter bound by optimizing over the whole set $\left\{z_{x}\right\}$ that appears in
Eq. (B13) instead of fixing all of them to be equal to obtain Eq. (19). However, the main use of the parameter $z$ in Eq. (B14) is to generalize the bound to an infinitesimal time step, as we do in Appendix D. 


\section{APPENDIX C: BOUND FOR THE PARALLEL STRATEGY}

In the parallel strategy, the action of $N$ channels is described as $\left\{\mathcal{E}_{\theta_{x}}^{\otimes N}\right\}$. The total QFI for the parallel strategy with $N$ uses is thus,

$$
\mathfrak{F}_{\mathbf{q}}^{N}:=\max _{\rho \in \mathcal{S}\left(\mathcal{H}_{S}^{\otimes N}\right)} \sum_{x=1}^{\mathfrak{p}} q_{x} \mathcal{F}\left(\mathcal{E}_{\theta_{x}}^{\otimes N}(\rho)\right) .
$$

The following result generalizes the analogous singleparameter one ([60] Theorem 5).

Theorem C.1: The total entanglement-assisted channel QFI for the parallel scheme with $N$ uses is upper bounded as follows:

$\mathfrak{F}_{\mathbf{q}}^{N} \leq 4 \min _{\mathfrak{h}}\left\{N\left\|\sum_{x=1}^{\mathfrak{p}} q_{x} \alpha_{x}\right\|+N(N-1)\left\|\sum_{i=1}^{\mathfrak{p}} q_{x} \beta_{x}^{2}\right\|\right\}$,

where $\alpha_{x}:=\partial_{\theta_{x}} \tilde{\mathbf{K}}_{x}^{\dagger} \partial_{\theta_{x}} \tilde{\mathbf{K}}_{x}$ and $\beta_{x}=\left(\partial_{\theta_{x}} \mathbf{K}_{x}-i h_{x} \mathbf{K}_{x}\right)^{\dagger} \mathbf{K}_{x}$.

Before proving this theorem, we need a simple inequality.

Lemma C.1: Given a collection of $\mathfrak{p}$ Hermitian matrices $A_{i}$, we have the following inequality between operator norms:

$$
\left\|\sum_{i=1}^{\mathfrak{p}} A_{i} \otimes A_{i}\right\| \leq\left\|\sum_{i=1}^{\mathfrak{p}} A_{i}^{2}\right\| \text {. }
$$

Proof.-We rewrite the initial matrix as follows:

$$
\sum_{i=1}^{\mathfrak{p}} A_{i} \otimes A_{i}=\sum_{i=1}^{\mathfrak{p}}\left(A_{i} \otimes \mathbb{1}\right)\left(\mathbb{1} \otimes A_{i}\right)=\tilde{A} \tilde{B}
$$

where $\quad \tilde{A}=\left[\begin{array}{c}A_{1} \otimes \mathbb{1} \\ \vdots \\ A_{\mathfrak{p}} \otimes \mathbb{1}\end{array}\right]^{\dagger}=\left[A_{1}, \ldots, A_{\mathfrak{p}}\right] \otimes \mathbb{1} \quad$ and
$\tilde{B}=\left[\begin{array}{c}\mathbb{1} \otimes A_{1} \\ \vdots \\ \mathbb{1} \otimes A_{\mathfrak{p}}\end{array}\right]=\mathbb{1} \otimes\left[\begin{array}{c}A_{1} \\ \vdots \\ A_{p}\end{array}\right]$. Now we can use the submultiplicativity of the operator norm

$$
\left\|\sum_{i=1}^{\mathfrak{p}} A_{i} \otimes A_{i}\right\|=\|\tilde{A} \tilde{B}\| \leq\|\tilde{A}\|\|\tilde{B}\|
$$

and obtain the desired result by noticing that $\|\tilde{A}\|=\sqrt{\left\|\tilde{A} \tilde{A}^{\dagger}\right\|}=\sqrt{\left\|\sum_{i}^{\mathfrak{p}} A_{i}^{2}\right\|}$ and $\|\tilde{B}\|=\sqrt{\|\tilde{B} \dagger \tilde{B}\|}=$ $\sqrt{\left\|\sum_{i}^{\mathfrak{p}} A_{i}^{2}\right\|}$.

We can now prove Theorem C.1, essentially following Ref. [60].

Proof of Theorem C.1.-The total entanglement-assisted channel QFI of the ensemble $\left\{\mathcal{E}_{\theta_{x}}^{\otimes N}\right\}$ is obtained using Theorem 1. Being a minimization over Kraus operators, if we restrict the minimization over operators with a particular form we obtain an upper bound. The most natural choice for the channels $\mathcal{E}_{\theta_{x}}^{\otimes N}$ is the tensor product of Kraus operators defined recursively

$$
\tilde{K}_{\mathbf{k}_{x}}^{(N+1)}=\tilde{K}_{k_{1}}^{(N)} \otimes \tilde{K}_{k_{2}}^{(1)},
$$

where $\mathbf{k}_{x} \in\left\{1, \ldots, r_{x}\right\}^{N} \times\left\{1, \ldots, r_{x}\right\}$ is a multi-index and $\tilde{\mathbf{K}}_{x}^{(1)}=\tilde{\mathbf{K}}_{x}$ are the Kraus operator of the original channel. Each multi-index $\mathbf{k}_{x}$ depends on $x$, yet for brevity, we suppress the dependence on $x$ when expressing its components $k_{i}:=k_{x, i}$. We introduce the quantities

$$
\alpha_{x}^{(N)}=\sum_{\mathbf{k}_{x}} \partial_{\theta_{x}} \tilde{K}_{\mathbf{k}_{x}}^{(N) \dagger} \partial_{\theta_{x}} \tilde{K}_{\mathbf{k}_{x}}^{(N)}
$$

$$
\beta_{x}^{(N)}=\sum_{\mathbf{k}_{x}} \partial_{\theta_{x}} \tilde{K}_{\mathbf{k}_{x}}^{(N) \dagger} \tilde{K}_{\mathbf{k}_{x}}^{(N)}
$$

and $\alpha_{x}:=\alpha_{x}^{(1)}$ and $\beta_{x}:=\beta_{x}^{(1)}$ are the quantities that appear in the statement of the theorem. Following the derivation of Ref. [60], we obtain

$$
\sum_{x=1}^{\mathfrak{p}} q_{x} \alpha_{x}^{(N)}=\sum_{x=1}^{\mathfrak{p}} q_{x}\left(\sum_{\substack{i, j \\ i+j=N-1}} \mathbb{1}^{\otimes i} \otimes \alpha_{x} \otimes \mathbb{1}^{\otimes j}-2 \sum_{\substack{i, j, k \\ i+j+k=N-2}} \mathbb{1}^{\otimes i} \otimes \beta_{x} \otimes \mathbb{1}^{\otimes j} \otimes \beta_{x} \otimes \mathbb{1}^{\otimes k}\right)
$$

the only difference being the additional summation over $x$. Thanks to the triangle inequality

$$
\left\|\sum_{x=1}^{\mathfrak{p}} q_{x} \alpha_{x}^{(N)}\right\| \leq N\left\|\sum_{x=1}^{\mathfrak{p}} q_{x} \alpha_{x}\right\|+N(N-1)\left\|\sum_{x=1}^{\mathfrak{p}} q_{x} \beta_{x} \otimes \beta_{x}\right\|,
$$

and the second term is upper bounded using Eq. (C3) to obtain Eq. (C2).
This bound is asymptotically equivalent to the adaptive bound when Heisenberg scaling is not allowed. From the triangle inequality for the operator norm, we also see that

$$
\begin{gathered}
4 \min _{\mathfrak{h}}\left\{N\left\|\sum_{x=1}^{\mathfrak{p}} q_{x} \alpha_{x}\right\|+N(N-1)\left\|\sum_{i=1}^{\mathfrak{p}} q_{x} \beta_{x}^{2}\right\|\right\} \\
\leq \sum_{x=1}^{\mathfrak{p}} q_{x} 4 \min _{h_{x}}\left\{N\left\|\alpha_{x}\right\|+N(N-1)\left\|\beta_{x}\right\|^{2}\right\},
\end{gathered}
$$


where the quantity on the right-hand side of Eq. (C11) is the sum of the independent single-parameter bounds; this is again a trivial bound that does not take into account inherent incompatibility.

\section{APPENDIX D: BOUND FOR A GENERAL MARKOVIAN NOISE MODEL}

We consider a probe system evolving in time according to a Gorini-Kossakowski-Lindblad-Sudarshan (GKLS) master equation:

$$
\begin{aligned}
\frac{d \rho}{d t}= & -i \theta_{x}\left[H_{x}, \rho\right]+\sum_{j=1}^{J_{x}} L_{x, j} \rho L_{x, j}^{\dagger} \\
& -\frac{1}{2}\left(L_{x, j}^{\dagger} L_{x, j} \rho+\rho L_{x, j}^{\dagger} L_{x, j}\right),
\end{aligned}
$$

where the parameter dependence enters linearly in the Hamiltonian part. We can derive a bound for the total QFI of the most general strategy, which includes the application of arbitrary fast and frequent control operations, by considering the channel $\mathcal{E}_{\theta_{x}, d t}$ obtained by integrating the master equation for a time $d t$ and taking the limit $d t \rightarrow 0$. For each channel, we choose the following $J_{x}+1$ Kraus operators that reproduce the dynamics up to first order in $d t$ :

$$
\begin{gathered}
K_{x, 0}=\mathbb{1}-\left(\frac{1}{2} \mathbf{L}_{x}^{\dagger} \mathbf{L}_{x}+i \theta_{x} H_{x}\right) d t+O\left(d t^{2}\right), \\
K_{x, j}=L_{x, j} \sqrt{d t}+O\left(d t^{\frac{3}{2}}\right), \quad j=1, \ldots, J_{x} .
\end{gathered}
$$

Given the structure of the Kraus operator, the Hermitian matrices in the minimization are written in the following block form:

$$
h_{x}=\left[\begin{array}{l|l}
h_{x}^{0} & \mathbf{h}_{x}^{\dagger} \\
\hline \mathbf{h}_{x} & \mathbb{\natural}_{x}
\end{array}\right] .
$$

With this choice, we can follow the same approach used in the single-parameter case $[4,5]$ and fix the total probing time $T$ such that the scheme is equivalent to a sequential one with a discrete number of channel uses $N=T / d t$ and eventually take the limit $d t \rightarrow 0$. The final result is obtained by using the infinitesimal Kraus operators (D2) in the sequential bound (B14), where the free parameter $z$ allows us to get a meaningful result for $d t \rightarrow 0$. In particular, we focus only on the case in which Heisenberg scaling $T^{2}$ is not possible and obtain the following SQL bound:

$$
\mathfrak{F}_{\mathbf{q}}^{T} \leq T \mathfrak{B}_{\mathbf{q}}, \quad \mathfrak{B}_{\mathbf{q}}:=4 \min _{\substack{\left\{\beta_{x}^{(1)}=0\right\} \\ \mathfrak{l}_{x}}}\left\|\sum_{x=1}^{\mathfrak{p}} q_{x} \alpha_{x}^{(1)}\right\|,
$$

where $\quad \alpha_{x}^{(1)}=\left(\mathbf{h}_{x}^{\left(\frac{1}{2}\right)} \mathbb{1}+\mathbb{h}_{x}^{(0)} \mathbf{L}_{x}\right)^{\dagger}\left(\mathbf{h}_{x}^{\left(\frac{1}{2}\right)} \mathbb{1}+\mathbb{h}_{x}^{(0)} \mathbf{L}_{x}\right), \quad \beta_{x}^{(1)}=$ $H_{x}+h_{x}^{0(1)} \mathbb{1}+\mathbf{h}_{x}^{\dagger\left(\frac{1}{2}\right)} \mathbf{L}_{x}+\mathbf{L}_{x}^{\dagger} \mathbf{h}_{x}^{\left(\frac{1}{2}\right)}+\mathbf{L}_{x}^{\dagger} h_{x}^{(0)} \mathbf{L}_{x}$, and the optimization variables are the set $\mathfrak{h}=\left\{h_{x}^{0(1)}, \mathbf{h}_{x}^{\left(\frac{1}{2}\right)}, \mathbb{l n}_{x}^{(0)}\right\}_{x=1}^{\mathfrak{p}}$. The subscript in brackets indicates the corresponding order in $d t$.

The conditions $\beta_{x}^{(1)}=0$ are known as "Hamiltonian in the Lindblad span" conditions, since they are equivalent to

$$
\begin{aligned}
& H_{x} \in \operatorname{span}_{\mathbb{R}}\left\{\mathbb{1},\left(L_{x, j}\right)^{\mathrm{H}}, i\left(L_{x, j}\right)^{\mathrm{AH}},\left(L_{x, j}^{\dagger} L_{x, j^{\prime}}\right)^{\mathrm{H}},\right. \\
& \left.\quad i\left(L_{x, j}^{\dagger} L_{x, j^{\prime}}\right)^{\mathrm{AH}}\right\},
\end{aligned}
$$

where $\mathrm{H}$ and $\mathrm{AH}$ denote the Hermitian and anti-Hermitian parts, and the sets are known as the Lindblad spans $[4,5,8,75]$ of the different GKLS master equations. These conditions have to be violated for all parameters in order to preserve Heisenberg scaling of the total variance with error correction [28].

\section{APPENDIX E: RECOVERING PREVIOUS RESULTS}

Here we show that a couple of existing results that have been derived in a different framework can be recovered as purification-based bounds.

\section{Unitary parameters}

The situation for unitary parameters is particularly simple, since there is only one Kraus operator. Kura and Ueda ([21] Theorem 1) have derived a general bound, and here we show that the same result can be obtained from the purification-based definition of the QFI matrix.

Corollary E.1: For noiseless multiparameter estimation with a linear parameter encoding $U_{\boldsymbol{\theta}}=e^{-i H_{\boldsymbol{\theta}}}=e^{-i \sum_{x=1}^{\mathfrak{p}} \theta_{x} G_{x}}$ with $\mathfrak{p}$ Hermitian generators $\left\{G_{x}\right\}_{x=1}^{\mathfrak{p}}$, we have

$$
\operatorname{tr} \mathcal{F}\left(U_{\boldsymbol{\theta}}\left|\psi_{0}\right\rangle\right) \leq \mathfrak{F} \leq 4\left\|\sum_{x=1}^{\mathfrak{p}} G_{x}^{2}\right\|
$$

Proof.-From Eq. (12) for a unitary evolution, we have

$\operatorname{tr} \mathcal{F}\left(U_{\boldsymbol{\theta}}\left|\psi_{0}\right\rangle\right) \leq 4 \min _{\mathfrak{h}}\left\|\sum_{x=1}^{\mathfrak{p}} \partial_{x} \tilde{U}_{\boldsymbol{\theta}}^{\dagger} \partial_{x} \tilde{U}_{\boldsymbol{\theta}}\right\| \leq 4\left\|\sum_{x=1}^{\mathfrak{p}} \partial_{x} U_{\boldsymbol{\theta}}^{\dagger} \partial_{x} U_{\boldsymbol{\theta}}\right\|$,

since the choice $h_{x}=0$ needs not be optimal. We recall the formula for the derivative of a unitary [11]

$$
\partial_{x} U_{\boldsymbol{\theta}}=-i U_{\boldsymbol{\theta}}\left(\int_{0}^{1} d \alpha e^{i \alpha H_{\theta}} G_{x} e^{-i \alpha H_{\theta}}\right) .
$$

We can use the triangle inequality for the integral to get the upper bound 


$$
\begin{aligned}
& \left\|\sum_{x=1}^{\mathfrak{p}} \partial_{x} U_{\boldsymbol{\theta}}^{\dagger} \partial_{x} U_{\boldsymbol{\theta}}\right\| \\
& =\left\|\sum_{x=1}^{\mathfrak{p}}\left(\int_{0}^{1} d \alpha e^{\mathrm{i} \alpha H_{\theta}} G_{x} e^{-i \alpha H_{\theta}}\right)\left(\int_{0}^{1} d \alpha^{\prime} e^{i \alpha^{\prime} H_{\theta}} G_{x} e^{-i \alpha^{\prime} H_{\theta}}\right)\right\| \\
& \leq \int_{0}^{1} d \alpha \int_{0}^{1} d \alpha^{\prime}\left\|\sum_{x=1}^{\mathfrak{p}}\left(e^{i \alpha H_{\theta}} G_{x} e^{-i \alpha H_{\theta}}\right)\left(e^{i \alpha^{\prime} H_{\theta}} G_{x} e^{-i \alpha^{\prime} H_{\theta}}\right)\right\| \\
& =\int_{0}^{1} d \alpha \int_{0}^{1} d \alpha^{\prime}\left\|\sum_{x=1}^{\mathfrak{p}} G_{x} e^{-i\left(\alpha-\alpha^{\prime}\right) H_{\theta}} G_{x}\right\| \\
& \leq \int_{0}^{1} d \alpha \int_{0}^{1} d \alpha^{\prime}\left\|e^{-i\left(\alpha-\alpha^{\prime}\right) H_{\theta}}\right\|\left\|\sum_{x=1}^{\mathfrak{p}} G_{x}^{2}\right\|=\left\|\sum_{x=1}^{\mathfrak{p}} G_{x}^{2}\right\|,
\end{aligned}
$$

where we use the inequality (B8).

In particular, when the parameters are frequencies $\omega_{i}=\theta_{i} / t$, we get $\mathfrak{F} \leq 4 t^{2}\left\|\sum_{x=1}^{\mathfrak{p}} G_{x}^{2}\right\|$ as in Ref. [21].

\section{Right logarithmic derivative channel bound}

Hayashi introduced a channel bound based on the right logarithmic derivative (RLD) QFI [126] for a singleparameter family of channels. Further properties and connections with hypothesis testing have been explored in Ref. [127]. While the applicability of this construction is more limited than general purification-based bounds [2], it has the advantage of being expressed only in terms of the Choi-Jamiołkowski (CJ) matrix of the channel. Very recently, the same approach has been extended to multiple parameters in Ref. [62]. Following the reasoning of Ref. [77], we show that, when it is defined, the multiparameter RLD channel bound corresponds to a particular purification, thus being less tight than the optimal bound.

The CJ matrix of the channel $\mathcal{E}_{\theta}: \mathcal{T}\left(\mathcal{H}_{\text {in }}\right) \rightarrow \mathcal{T}\left(\mathcal{H}_{\text {out }}\right)$ is an unnormalized state on the space $\mathcal{T}\left(\mathcal{H}_{\text {out }} \otimes \mathcal{H}_{A}\right)$, where $\operatorname{dim} \mathcal{H}_{A}=d_{\text {out }}$ is defined as

$$
\Omega_{\boldsymbol{\theta}}=\mathcal{E}_{\boldsymbol{\theta}} \otimes \mathcal{I}(|\mathbb{1}\rangle\langle\mathbb{1}|)=\sum_{i j} \mathcal{E}_{\boldsymbol{\theta}}\left(| i \rangle \langle j | _ { S } ) \otimes | i \rangle \left\langle\left.j\right|_{A},\right.\right.
$$

where $\left\{|j\rangle_{S}\right\}_{j=1}^{d_{\text {in }}}$ and $\left\{|j\rangle_{A}\right\}_{j=1}^{d_{\text {out }}}$ are orthonormal bases of $\mathcal{H}_{\text {in }}$ and $\mathcal{H}_{A}$, while $|\mathbb{1}\rangle=\sum_{i=1}^{d_{\text {in }}}|i\rangle_{S}|i\rangle_{A}$ is an unnormalized maximally entangled state. We use the compact notation $|M\rangle=\sum_{i, j=1}^{d_{\text {in }}}\langle i|M| j\rangle|i\rangle|j\rangle=M \otimes \mathbb{1}|\mathbb{1}\rangle=\mathbb{1} \otimes M^{T}|\mathbb{1}\rangle$ that can be used to write the CJ matrix as $\Omega_{\theta}=$ $\sum_{i=1}^{l}\left|K_{i}\right\rangle\left\langle K_{i}\right|$ where the operators $K_{i}$ are an arbitrary Kraus decomposition of the channel. The CJ matrix can be diagonalized as $\Omega_{\boldsymbol{\theta}}=\sum_{i=1}^{r} \lambda_{i}\left|\Psi_{i}\right\rangle\left\langle\Psi_{i}\right|$, where $\lambda_{i}>0$ and $\left\langle\Psi_{i} \mid \Psi_{j}\right\rangle=\delta_{i j}$ and this defines the canonical Kraus decomposition $\left|K_{i}\right\rangle=\sqrt{\lambda_{i}}\left|\Psi_{i}\right\rangle$.

The following bound for a general adaptive strategy with $N$ uses of the channel was obtained in Ref. [62] (we consider $W=\mathbb{1}$ without loss of generality, since it can be understood as a reparametrization)

$$
\begin{gathered}
\Delta^{2} \tilde{\boldsymbol{\theta}} \geq \frac{\mathfrak{p}^{2}}{N \mathfrak{B}^{R}} \\
\mathfrak{B}^{R}=\left\|\sum_{x=1}^{\mathfrak{p}} \operatorname{Tr}_{A}\left[\left(\partial_{x} \Omega_{\boldsymbol{\theta}}\right) \Omega_{\boldsymbol{\theta}}^{-1}\left(\partial_{x} \Omega_{\boldsymbol{\theta}}\right)\right]\right\|
\end{gathered}
$$

valid when the following finiteness condition holds:

$$
\sum_{x=1}^{\mathfrak{p}}\left(\partial_{x} \Omega_{\boldsymbol{\theta}}\right)^{2} \Pi_{\Omega}^{\perp}=0
$$

where $\Pi_{\Omega}^{\perp}$ is the projector on the kernel of the CJ matrix $\Omega_{\boldsymbol{\theta}}$ and the inverse is taken on the support. Otherwise, the bound is trivial (diverging) when Eq. (E8) is not satisfied. In a moment, we show that this condition is equivalent to the following conditions:

$$
\begin{aligned}
\partial_{x} K_{i} & =\sum_{j} \nu_{x, i j} K_{i} \Rightarrow \\
\partial_{x} \Omega_{\boldsymbol{\theta}} & =\sum_{i j} \mu_{x, i j}\left|K_{i}\right\rangle\left\langle K_{j}\right| \quad \forall x=1, \ldots, \mathfrak{p},
\end{aligned}
$$

where $\left\{\nu_{x}\right\}$ are $\mathfrak{p}$ complex matrices, $\mu_{x}=\nu_{x}+\nu_{x}^{\dagger}$ are twice their Hermitian part, and $\left\{K_{i}\right\}$ is the canonical Kraus representation. This also means that the partial derivatives of the CJ matrix vanish outside the support of the CJ matrix. For a single-parameter channel, this condition is known as $\varphi$ nonextremality [2,77]. When it is satisfied for all parameters of a quantum channel, we dub it local nonextremality, or equivalently, we say the channel is locally nonextremal. Now we proceed to show the equivalence of local nonextremality (E9) and the finiteness condition (E8). The situation is essentially equivalent to the single-parameter case described in Ref. [77], modulo minor observations.

When the channel is locally nonextremal (E9) the finiteness condition (E8) holds trivially, since $\left\langle K_{i}\right| P_{\Omega}^{\perp}=0 \quad \forall i$. On the other hand, we notice that the condition (E8) is equivalent to

$$
\Pi_{\Omega}^{\perp}\left[\sum_{x=1}^{\mathfrak{p}}\left(\partial_{x} \Omega_{\boldsymbol{\theta}}\right)^{2}\right] \Pi_{\Omega}^{\perp}=0
$$

since the matrix $\sum_{x=1}^{\mathfrak{p}}\left(\partial_{x} \Omega_{\boldsymbol{\theta}}\right)^{2}$ is Hermitian. We write the derivatives of the Kraus operators separating the components in the support of $\Omega_{\theta}$ and those in the kernel: $\left|\partial_{x} K_{i}\right\rangle=\sum_{j=1}^{r} \nu_{x, i j}\left|K_{j}\right\rangle+\left|L_{x, i}\right\rangle$, where $\left\langle K_{i} \mid L_{x, j}\right\rangle=0 \forall i$, $j=1, \ldots, r \forall x=1, \ldots, \mathfrak{p}$ and $\left\{\nu_{x}\right\}$ are $\mathfrak{p}$ complex matrices of dimension $r \times r$. The condition (E10) becomes 


$$
\sum_{x=1}^{\mathfrak{p}}\left(\sum_{i=1}^{r}\left|L_{x, i}\right\rangle\left\langle K_{i}\right|\right)\left(\sum_{j=1}^{r}\left|K_{j}\right\rangle\left\langle L_{x, i}\right|\right)=0 .
$$

This equality has the form $\sum_{i} A_{i}^{\dagger} A_{i}=0$, and since $A_{i}^{\dagger} A_{i} \geq 0$ we must have that $A_{i}=0 \forall i$; furthermore, since the vectors $\left|K_{i}\right\rangle$ are orthogonal, we obtain $\left|L_{x, i}\right\rangle=0 \forall i, x$, which means

$$
\partial_{x} K_{i}=\sum_{j} \nu_{x, i j} K_{i}, \quad \forall x=1, \ldots, \mathfrak{p} .
$$

From this, we have that

$$
\begin{aligned}
\partial_{x} \Omega_{\boldsymbol{\theta}} & =\sum_{i=1}^{r}\left|\partial_{x} K_{i}\right\rangle\left\langle K_{i}|+| K_{i}\right\rangle\left\langle\partial_{x} K_{i}\right| \\
& =\sum_{i, j=1}^{r} \nu_{x, i j}\left|K_{j}\right\rangle\left\langle K_{i}\left|+\nu_{x, i j}^{*}\right| K_{i}\right\rangle\left\langle K_{j}\right| \\
& =\sum_{i, j=1}^{r} \mu_{x, i j}\left|K_{j}\right\rangle\left\langle K_{i}\right|,
\end{aligned}
$$

where $\mu_{x}=\nu_{x}+\nu_{x}^{\dagger}$ is twice the Hermitian part.

Now we can show that Eq. (E9) implies the satisfaction of the HKS condition for all parameters and that the bound (E6) has the same form $4\left\|\sum_{x} \alpha_{x}\right\|$ of purification-based bounds. From the derivatives of the Kraus operators (E9), we obtain the equality

$$
\begin{aligned}
& \frac{i}{2} \sum_{i}\left|K_{i}\right\rangle\left\langle\partial_{x} K_{i}|-| \partial_{x} K_{i}\right\rangle\left\langle K_{i}\right| \\
& =-\frac{i}{2} \sum_{i, j}\left(\nu_{x}-\nu_{x \dagger}\right)_{i j}\left|K_{i}\right\rangle\left\langle K_{j}\left|=\sum_{i, j}\left(h_{x}^{R}\right)_{i j}\right| K_{i}\right\rangle\left\langle K_{j}\right|,
\end{aligned}
$$

where $h_{x}^{R}=-i \nu_{x}^{A}=-(i / 2)\left(\nu^{x}-\nu^{x_{\dagger}^{\dagger}}\right)$, and by partial tracing over $\mathcal{H}_{A}$ we obtain the HKS conditions $\partial_{x} \mathbf{K}^{\dagger} \mathbf{K}=-i \mathbf{K}^{\dagger} h_{x}^{R} \mathbf{K}$, since $\partial_{x} \mathbf{K}^{\dagger} \mathbf{K}=-\mathbf{K}^{\dagger} \partial_{x} \mathbf{K}$. Since $\left\langle K_{i}\left|\Omega_{\boldsymbol{\theta}}^{-1}\right| K_{j}\right\rangle=\delta_{i j}$, the operators appearing inside the summation in Eq. (E6) can be written as follows:

$$
\begin{aligned}
\operatorname{Tr}_{A}\left[\left(\partial_{x} \Omega_{\boldsymbol{\theta}}\right) \Omega_{\boldsymbol{\theta}}^{-1}\left(\partial_{x} \Omega_{\boldsymbol{\theta}}\right)\right] & =\operatorname{Tr}_{A}\left[\sum_{i, j}\left(\mu_{x}^{2}\right)_{i j}\left|K_{j}\right\rangle\left\langle K_{i}\right|\right] \\
& =4 \partial_{x} \tilde{\mathbf{K}}^{\dagger} \partial_{x} \tilde{\mathbf{K}},
\end{aligned}
$$

where $\partial \tilde{\mathbf{K}}=\partial_{x} \mathbf{K}-i h_{x}^{R} \mathbf{K}$, since $\partial_{x} \mathbf{K}=\nu_{x} \mathbf{K}$ from Eq. (E9) and $\nu_{x}-i h_{x}^{R}=\frac{1}{2} \mu^{x}$ by definition. Therefore, we show that the bound (E6) has the same form of the bound (20), but it is evaluated with a generally suboptimal choice $\left\{h_{x}^{R}\right\}_{x=1}^{\mathfrak{p}}$, implying that

$$
\mathfrak{B} \leq \mathfrak{B}^{R} .
$$

\section{APPENDIX F: SEMIDEFINITE PROGRAMS FOR THE BOUNDS}

We introduce the following matrix:

$$
\mathbf{D}=\left[\begin{array}{c}
\sqrt{q_{1}}\left(\partial_{\theta_{1}} \mathbf{K}_{1}-i h_{1} \mathbf{K}_{1}\right) \\
\vdots \\
\sqrt{q_{\mathfrak{p}}}\left(\partial_{\theta_{\mathfrak{p}}} \mathbf{K}_{\mathfrak{p}}-i h_{\mathfrak{p}} \mathbf{K}_{\mathfrak{p}}\right)
\end{array}\right],
$$

where the derivatives of the Kraus operator of each channel $\mathcal{E}_{\theta_{x}}$ are put in a column. This matrix has dimension $\bar{d} \times d_{\text {in }}$, where $\bar{d}=d_{\text {out }} \sum_{x=1}^{\mathcal{p}} r_{x}$, where $r_{x}$ is the number of Kraus operators of each channel. When considering the multiparameter estimation scenario with a single channel with $r$ Kraus operators, we have $\bar{d}=\mathfrak{p} d_{\text {out }} r$.

The bound for a single use of the channel (12) can be rewritten as the following SDP:

$$
\mathfrak{F}=4 \min _{t,\left\{h_{x}\right\}} t \quad \text { subject to }\left[\begin{array}{cc}
t \mathbb{1}_{d_{\text {in }}} & \mathbf{D}^{\dagger} \\
\mathbf{D} & \mathbb{1}_{\bar{d}}
\end{array}\right] \geq 0 .
$$

The finite- $N$ bound (C2) for the parallel strategy, i.e., $\mathfrak{B}_{N}=4 \min _{\mathfrak{h}}\left\{\left\|\sum_{x=1}^{\mathfrak{p}} q_{x} \alpha_{x}\right\|+(N-1)\left\|\sum_{i=1}^{\mathfrak{p}} q_{x} \beta_{x}^{2}\right\|\right\}$, can be obtained similarly to the single-parameter case [72] as follows:

$$
\begin{gathered}
\mathfrak{B}_{N}=4 \min _{t, v,\left\{h_{x}\right\}}\{t+(N-1) v\} \\
\text { subject to }\left[\begin{array}{cc}
t \mathbb{1}_{d_{\text {in }}} & \mathbf{D}^{\dagger} \\
\mathbf{D} & \mathbb{1}_{\bar{d}}
\end{array}\right] \geq 0\left[\begin{array}{cc}
v \mathbb{1}_{d_{\text {in }}} & \mathbf{B}^{\dagger} \\
\mathbf{B} & \mathbb{1}_{\bar{d}}
\end{array}\right] \geq 0,
\end{gathered}
$$

where we introduce

$$
\mathbf{B}=\left[\begin{array}{c}
\sqrt{q_{1}}\left(\partial_{\theta_{1}} \mathbf{K}_{1}-i h_{1} \mathbf{K}_{1}\right)^{\dagger} \mathbf{K}_{1} \\
\vdots \\
\sqrt{q_{\mathfrak{p}}}\left(\partial_{\theta_{\mathfrak{p}}} \mathbf{K}_{\mathfrak{p}}-i h_{\mathfrak{p}} \mathbf{K}_{\mathfrak{p}}\right)^{\dagger} \mathbf{K}_{\mathfrak{p}}
\end{array}\right] .
$$

The asymptotic SQL bound (20) is obtained simply by imposing the additional linear constraints $\beta_{x}=0 \forall x=$ $1, \ldots, \mathfrak{p}$ to the SDP (F2).

Finally, the asymptotic SQL bound for Markovian noise (D5) is obtained in the same way, but using

$$
\mathbf{D}_{\text {Mark }}=\left[\begin{array}{c}
\sqrt{q_{1}}\left(\mathbf{h}_{1}^{\left(\frac{1}{2}\right)} \mathbb{1}+\mathbb{h}_{1}^{(0)} \mathbf{L}_{1}\right) \\
\vdots \\
\sqrt{q_{2}}\left(\mathbf{h}_{\mathfrak{p}}^{\left(\frac{1}{2}\right)} \mathbb{1}+\mathbb{l}_{\mathfrak{p}}^{(0)} \mathbf{L}_{\mathfrak{p}}\right)
\end{array}\right]
$$

instead of $\mathbf{D}$; here, $d_{\text {in }}=d_{\text {out }}=d, \bar{d}=d \sum_{x=1}^{\mathfrak{p}} J_{x}$ (where $J_{x}$ is the number of collapse operators of each master 
equation), and the optimization runs over the Hermitian matrices $\left\{h_{x}^{0(1)}, \mathbf{h}_{x}^{\left(\frac{1}{2}\right)}, \mathfrak{l}_{x}^{(0)}\right\}_{x=1}^{\mathfrak{p}}$ with the linear constraints $\beta_{x}^{(1)}=0$.

\section{APPENDIX G: ALGORITHM TO FIND AN OPTIMAL STATE AND EVALUATE ITS QFI MATRIX}

The derivation of the analogous single-parameter algorithm [7,8] relies on Sion's minimax theorem and remains unchanged for our multiparameter figure of merit. The only difference is that now we have a collection of $\mathfrak{p}$ matrices $\mathfrak{h}=\left\{h_{x}\right\}_{x=1}^{\mathfrak{p}}$ instead of just one. Thus, we can adapt the two-step procedure of Appendix 8 in Ref. [8]; the algorithm to find an optimal state goes as follows (for simplicity, we introduce the operator $\bar{\alpha}=\sum_{x=1}^{\mathfrak{p}} q_{x} \alpha_{x}$ ).

(1) Find a set of optimal Hermitian matrices $\mathfrak{h}^{\star}$ by solving the SDP (F2), such that the operator $\bar{\alpha}^{\star}=$ $\left.\bar{\alpha}\right|_{\mathfrak{h}=\mathfrak{h}^{\star}}$ satisfies $\min _{\mathfrak{h}}\|\bar{\alpha}\|=\left\|\bar{\alpha}^{\star}\right\|$.

(2) The support of the optimal state $\rho^{\star}$ is the eigenspace of the largest eigenvalue of the operator $\bar{\alpha}^{\star}$ and $\forall x$, and it satisfies the constraints

$$
\begin{array}{r}
\operatorname{Re}\left\{\operatorname{Tr}\left[\rho^{\star}\left(i \mathbf{K}_{x}^{\dagger} \Delta h_{x}\right)\left(\partial_{x} \mathbf{K}_{x}-i h_{x} \mathbf{K}\right)\right]\right\}=0, \\
\forall \Delta h_{x} \in \mathbb{C}_{r_{x} \times r_{x}}, \quad \Delta h_{x}=\left(\Delta h_{x}\right)^{\dagger} .
\end{array}
$$

The constraints (G1) are linear constraints on $\rho^{\star}$, and in practice they are imposed by fixing a basis of $r_{x} \times r_{x}$ Hermitian matrices. We remark that the optimal state is generally mixed when the largest eigenvalue of $\bar{\alpha}^{\star}$ has multiplicity greater than 1 . When a mixed state is optimal, it means that an optimal strategy is to use an extended channel and take advantage of entanglement with the auxiliary system, as it is clear from the proof of Theorem 1.

This algorithm allows one to find an optimal state attaining the total QFI, even when it corresponds to a random sensing scenario and the Kraus operators $\mathbf{K}_{x}$ pertain to different quantum channels. If we work in the multiparameter estimation scenario, there is only one vector $\mathbf{K}$ of Kraus operators; once an optimal state is found, the QFI matrix elements are evaluated as

$$
\mathcal{F}_{x y}=4 \operatorname{Re}\left\{\operatorname{Tr}\left[\rho^{\star}\left(\partial_{x} \mathbf{K}-i h_{x}^{\star} \mathbf{K}\right)^{\dagger}\left(\partial_{y} \mathbf{K}-i h_{y}^{\star} \mathbf{K}\right)\right]\right\},
$$

since the matrices $\mathfrak{h}^{\star}$ correspond to the optimal purification, we can apply the purification-based definition (24).

The same algorithm can be adapted to the asymptotic case by solving the SDP with the constraints $\beta_{x}=0$ and additionally imposing $\mathbf{K}_{x}^{\dagger} \Delta h_{x} \mathbf{K}_{x}=0 \forall x$. In practice, these constraints are imposed by using a basis of the null space of the map $h \mapsto \sum_{j, k=1}^{r_{x}} h_{j k} K_{x, j}^{\dagger} K_{x, k}$ from $r_{x} \times r_{x}$ to $d_{\text {in }} \times d_{\text {in }}$ Hermitian matrices.

\section{APPENDIX H: DETAILS ON THE EVALUATION OF THE BOUNDS FOR QUANTUM-METROLOGY APPLICATIONS}

In the following calculations, we make a series of Ansätze on the form of the optimal matrices $\mathfrak{h}$. These are mostly inspired by the numerical solution and justified by symmetry arguments. While the optimality of the presented solutions is tested against numerical results, we remark that any allowed choice of $\mathfrak{h}$ provides a valid bound on the total QFI. To ease the notation, in this section we move the parameter label $x$ of the matrices $\mathfrak{h}$ to the superscript when necessary.

\section{Generalized amplitude damping channel}

The generalized amplitude damping channel is a qubit channel with the following Kraus operators [128]:

$$
\begin{aligned}
& K_{0}=\sqrt{1-\nu}\left[\begin{array}{cc}
1 & 0 \\
0 & \sqrt{1-\gamma}
\end{array}\right], \quad K_{1}=\sqrt{1-\nu}\left[\begin{array}{cc}
0 & \sqrt{\gamma} \\
0 & 0
\end{array}\right], \\
& K_{2}=\sqrt{\nu}\left[\begin{array}{cc}
\sqrt{1-\gamma} & 0 \\
0 & 1
\end{array}\right], \quad K_{3}=\sqrt{\nu}\left[\begin{array}{cc}
0 & 0 \\
\sqrt{\gamma} & 0
\end{array}\right], \quad(\mathrm{H} 1)
\end{aligned}
$$

and we are interested in the estimation of both the parameters $\nu$ and $\gamma$. The estimation of $\gamma$ was studied in great detail in Ref. [129]. The multiparameter problem was studied in Ref. [62] as an application of the RLD channel bound introduced in Appendix E2.

For this model, sequential or parallel strategies do not give any advantage and we observe $\mathfrak{F}=\mathfrak{B}$ and $\mathfrak{I}=\mathfrak{I}_{\infty}$. The only nonzero elements of the optimal purification matrices are $h_{02}^{\nu}=h_{02}^{\nu *}=i A$ and $h_{02}^{\gamma}=h_{02}^{\gamma *}=i B$, where $A$ and $B$ are real numbers, but we do not report the full details to find $A$ and $B$ as functions of $\gamma$ and $\nu$. However, it is simple to check this statement numerically by solving the SDP; the code for this example can be found in Ref. [73]. We mention that the optimal scheme is to use a probe state $\sqrt{a}|00\rangle+\sqrt{1-a}|11\rangle$, making use of an auxiliary system, i.e., considering the extended channel $\mathcal{E}_{\nu, \gamma} \otimes \mathcal{I}$. The optimal degree of entanglement $a$ with the auxiliary system depends on the parameter values via a rather complicated function.

Qualitatively, the probe incompatibility cost is a decreasing function of $\gamma$ for a fixed $\nu \neq \frac{1}{2}$. Moreover, the problem is symmetrical around the value $\nu=\frac{1}{2}$, and incompatibility decreases symmetrically as $\nu$ goes from the extremes 0 and 1 to $\frac{1}{2}$ and only for $\eta=\frac{1}{2}$ there is no probe incompatibility.

For this problem, it is interesting to compare our result to the RLD bound, already evaluated in Ref. [62] Appendix F. One can see immediately that the multiparameter RLD is not tight for this problem, and it does not detect any probe incompatibility since it can be easily checked that

$$
\mathfrak{B}^{R}=\mathfrak{B}_{\gamma}^{R}+\mathfrak{B}_{\nu}^{R} .
$$


To give an idea, we report numerical results for a particular choice of parameters $(\nu, \gamma)=\left(\frac{1}{4}, \frac{1}{2}\right)$ for which we obtain $\mathfrak{B}^{R} \approx 10.67>\mathfrak{F}_{\nu}+\mathfrak{F}_{\gamma} \approx 4.72>\mathfrak{F} \approx 3.84$.

\section{Hamiltonian tomography with erasure noise}

\section{a. Diagonal generators: Lossy multiphase estimation}

With the Kraus operators (29), we obtain

$$
K_{i}^{\dagger} K_{j}= \begin{cases}(1-\eta)|i\rangle\langle j|, & i, j>0, \\ \eta \mathbb{1}_{d}, & j=i=0,\end{cases}
$$

and the $\beta_{x}=0$ HKS conditions become

$$
-|x\rangle\left\langle x\left|=h_{00}^{x} \eta \mathbb{1}+(1-\eta) \sum_{i j} h_{i j}^{x}\right| i\right\rangle\langle j|,
$$

which entail

$$
\begin{aligned}
& h_{00}^{x} \eta+h_{i i}^{x}(1-\eta)=0, \quad i \neq x, \\
& h_{00}^{x} \eta+h_{x x}^{x}(1-\eta)=-1, \\
& h_{i j}^{x}(1-\eta)=0, \quad i \neq j .
\end{aligned}
$$

Therefore, taking advantage of the symmetry of the problem, we can parametrize the matrices $h^{x}$ satisfying the constraint as follows:

$$
\begin{gathered}
h_{00}^{x}=A, \quad h_{x x}^{x}=-\frac{1+A \eta}{1-\eta}, \\
h_{i i}^{x}=-\frac{A \eta}{1-\eta}, \quad h_{i j}^{x}=0, h_{0 i}^{x}=c_{i},
\end{gathered}
$$

but we simplify the calculation with the Ansatz $c_{i}=0$. We use the simplified form (27) to compute the bound. We obtain

$$
\begin{array}{r}
H=\sum_{x=1}^{\mathfrak{p}}\left(h^{x}\right)^{2}=\operatorname{diag}(X, Y, Y, \ldots, Y), \\
X=\mathfrak{p} A^{2}, \quad Y=\frac{(\mathfrak{p}-1) A^{2} \eta^{2}+(1+A \eta)^{2}}{(1-\eta)^{2}},
\end{array}
$$

and since $\sum_{x=1}^{\mathfrak{p}} G_{x}^{2}=\mathbb{1}$, we have the function

$$
\left\|-\mathbb{1}+\sum_{i, j=0}^{d} H_{i j} K_{i}^{\dagger} K_{j}\right\|=-1+X \eta+(1-\eta) Y,
$$

which is minimized for $A=-1 / \mathfrak{p}$, giving the bound $\mathfrak{B}_{\text {diag }}=[\eta /(1-\eta)][4 \mathfrak{p} /(\mathfrak{p}-1)]$.

The previous calculation is obtained with $\mathfrak{p}=d \geq 2$, but we can repeat the same calculation with $d>\mathfrak{p} \geq 2$ and keep the same diagonal matrices $h^{x}$ in Eq. (H6) as before, obtaining now
$H^{\prime}=\sum_{x=1}^{\mathfrak{p}}\left(h^{x}\right)^{2}=\operatorname{diag}(X, Y, Y, \ldots, Y, Z, \ldots, Z)$,

$$
Z=\frac{\mathfrak{p} A^{2} \eta^{2}}{(1-\eta)^{2}}
$$

where $Y$ is repeated $\mathfrak{p}$ times and $Z$ is repeated $d-\mathfrak{p}$ times. The operator inside the norm now has one block $[-1+X \eta+(1-\eta) Y] \mathbb{1}_{\mathfrak{p}}$ analogous to the previous one [Eq. (H10)] and another block $[Z(1-\eta)+\eta X] \mathbb{1}_{d-p}$, where $\mathbb{1}_{\mathfrak{p}}$ is the projector on the span of the first $\mathfrak{p}$ canonical vectors and $\mathbb{1}_{d-\mathfrak{p}}=\mathbb{1}-\mathbb{1}_{\mathfrak{p}}$. The minimization of the operator norm produces the same result as in the previous case, since the optimal maximal eigenvalue always pertains to the first block.

\section{b. Off-diagonal generators}

We focus on the $d(d-1) / 2$ real off-diagonal generators $G_{\mu \nu}=\frac{1}{2}(|\mu\rangle\langle\nu|+| \mu\rangle\langle\nu|)$, and we use the convention $\mu>\nu$. The HKS conditions entail

$-\frac{1}{2}(|\mu\rangle\langle\nu|+| \nu\rangle\langle\mu|)=h_{00}^{\mu \nu} \eta \mathbb{1}+(1-\eta) \sum_{i, j>0} h_{i j}^{\mu \nu}|i\rangle\langle j|$,

and for $i, j>0$ we have

$h_{i j}^{\mu \nu}=-\frac{1}{2(1-\eta)}\left(\delta_{i \mu} \delta_{j \nu}+\delta_{i \nu} \delta_{j \mu}\right), \quad i \neq j$,

$h_{i i}^{\mu \nu}=-\frac{\eta}{1-\eta} h_{00}^{\mu \nu}, \quad i=j$.

We assume the following form:

$$
\begin{aligned}
h_{00}^{\mu \nu} & =A, \quad h_{i 0}^{\mu \nu}=0 \forall i>0, \\
h_{i j}^{\mu \nu} & =-\frac{\eta}{1-\eta} A \delta_{i j}-\frac{1}{2(1-\eta)}\left(\delta_{i \mu} \delta_{j \nu}+\delta_{i \nu} \delta_{j \mu}\right),
\end{aligned}
$$

from which we obtain

$$
\begin{aligned}
& \sum_{\mu>\nu}\left(h^{\mu \nu}\right)^{2} \\
& =\frac{d(d-1) A^{2}}{2}|0\rangle\left\langle 0\left|+\frac{d-1}{4(1-\eta)^{2}}\left(1+2 d \eta^{2} A^{2}\right) \sum_{i=1}^{d}\right| i\right\rangle\langle i| \\
& \quad+\frac{\eta A}{(1-\eta)^{2}} \sum_{\mu>\nu}(|\mu\rangle\langle\nu|+| \nu\rangle\langle\mu|) .
\end{aligned}
$$

This is a spherical model (according to the terminology of Ref. [21]): $\sum_{\mu>\nu}\left(G_{\mu \nu}\right)^{2}=[(d-1) / 4] \mathbb{1}$, and the function to minimize in the bound (27) becomes 


$$
\| \frac{d-1}{4} \frac{\eta}{1-\eta} \mathbb{1}+\frac{d(d-1) A^{2}}{2} \frac{\eta}{1-\eta} \mathbb{1}+\frac{\eta A}{1-\eta} \sum_{i \neq j}|i\rangle\langle j| \|,
$$

and the optimal choice is simply $A=0$ so the bound is $\mathfrak{B}_{\text {real }}=[\eta /(1-\eta)](d-1)$. For the imaginary off-diagonal elements, we can repeat the same reasoning and we arrive at the same bound.

We notice that the three operators $\sum_{x} \alpha_{x}$ for the three submodels are all proportional to the identity, and so they saturate the triangle inequality with equality as per Eq. (30).

\section{Phase and loss}

First, we consider the two separate single-parameter problems. For phase estimation, we have the single-use bound $\mathfrak{F}_{\theta}=\left[4 \eta /(\sqrt{\eta}+1)^{2}\right]$ corresponding to the optimal matrix $h_{\theta}=\operatorname{diag}\{-1+[1 /(1+\sqrt{\eta})],-1\}$ and the asymptotic bound $\mathfrak{B}_{\theta}=[4 \eta /(1-\eta)]$ corresponding to the matrix $h_{\theta}=\operatorname{diag}\{0,[-1 /(1-\eta)]\}$. For the estimation of $\eta$, both bounds coincide and we have $\mathfrak{F}_{\eta}=\mathfrak{B}_{\eta}=\{1 /[\eta(1-\eta)]\}$, and the optimal $h_{\eta}=0$ means that the original Kraus operators are already the optimal purification.

The single-use incompatibility cost (9) requires solving the following minimization:

$$
\min _{h_{\theta}, h_{\eta}}\left\|\frac{1}{\mathfrak{F}_{\theta}} \alpha_{\theta}+\frac{1}{\mathfrak{F}_{\eta}} \alpha_{\eta}\right\|,
$$

and the optimal matrices are $h_{\theta}=\operatorname{diag}\left\{-\left[\left(\eta^{3 / 2}-\right.\right.\right.$ $\left.\left.\left.\sqrt{2} \sqrt{\eta^{3 / 2}+\eta}+\eta\right) /\left(\eta^{3 / 2}+\eta-\sqrt{\eta}-1\right)\right],-1\right\} \quad$ and $h_{\eta}=0$, from which we obtain Eq. (35).

For the asymptotic incompatibility bound, the condition $\beta_{\theta}=0$ constrains the matrix $h_{\theta}$ to be the same as in the single-parameter case. We obtain $4 \alpha_{\theta}=\operatorname{diag}\left(\mathfrak{B}_{\theta}, 0\right)$ and $4 \alpha_{\eta}=\operatorname{diag}\left(\mathfrak{B}_{\eta}, 0\right)$, from which we can clearly see that there is no probe incompatibility: $\mathfrak{J}_{\infty}=1$ and $\mathfrak{B}=\mathfrak{B}_{\theta}+\mathfrak{B}_{\eta}$.

If we consider a description of the problem in terms of modes (instead of formally treating the photons as distinguishable particles), the evolution is described by a singlemode channel with the following Kraus operators:

$$
K_{l}=\sqrt{\frac{(1-\eta)^{l}}{l !}} e^{-i \theta a^{\dagger} a} \sqrt{\eta^{a^{\dagger}} a} a^{l}, \quad l=1, \ldots, N,
$$

where $N$ is the total photon number, and we use bosonic operators $a=\sum_{j=0}^{\infty} \sqrt{j+1}|j\rangle\langle j+1|$. The single-use bound for this channel is equivalent to an optimization of the total QFI over two-mode states of the form $\sum_{m=0}^{N} \psi_{m}|m, M-m\rangle$. We evaluate our probe incompatibility measure for these finite- $N$ channels, and we find that it decreases as $N$ increases, supporting our finding that it should disappear asymptotically. For example, going from $N=2$ to $N=20$, we find that probe incompatibility decreases from the value 1.0829 to 1.0712 (approximately
$1 \%$ ) for $\eta=0.05$, from 1.1726 to 1.1392 (approximately

$3 \%$ ) for $\eta=0.5$, and from 1.2703 to 1.1952 (approximately $6 \%$ ) for $\eta=0.95$.

\section{Phase and dephasing}

This problem is particularly simple, since the optimal matrices are always identical to the single-parameter ones. Regarding the parameter $\phi$, we have the single-use bound [72] $\mathfrak{\mho}_{\theta}=\eta^{2}$ corresponding to $h_{\theta}=\frac{1}{2} \mathbb{1}+\left[\left(\sqrt{1-\eta^{2}}\right) / 2\right] \sigma_{x}$ and the asymptotic bound [2] $\mathfrak{B}_{\theta}=\left[\eta^{2} /\left(1-\eta^{2}\right)\right]$ corresponding to $h_{\theta}=\frac{1}{2} \mathbb{1}+\left[1 /\left(2 \sqrt{1-\eta^{2}}\right)\right] \sigma_{x}$. Analogous to loss estimation in the previous section, the Kraus operators are already optimal for estimating $\eta$, i.e., $h_{\eta}=0$, and we have $\mathfrak{\Im}_{\eta}=\mathfrak{B}_{\eta}=\left[1 /\left(1-\eta^{2}\right)\right]$.

For these optimal matrices, we obtain $4 \alpha_{\theta}=\mathfrak{F}_{\theta} \mathbb{1}$ for a single use (and analogous $4 \alpha_{\theta}=\mathfrak{B}_{\theta} \mathbb{1}$ for the asymptotic case) and $4 \alpha_{\eta}=\mathfrak{F}_{\eta} \rrbracket$. Therefore, we see no probe incompatibility $\mathfrak{I}=\mathfrak{I}_{\infty}=1, \mathfrak{F}=\mathfrak{F}_{\theta}+\mathfrak{F}_{\eta}$, and $\mathfrak{B}=\mathfrak{B}_{\theta}+\mathfrak{B}_{\eta}$.

\section{Diagonal generators with qudit dephasing}

We can use the following Kraus representation for the qudit dephasing channel:

$K_{0}=\sqrt{\eta} \mathbb{1}, \quad K_{j}=\sqrt{1-\eta}|k\rangle\langle k| i=1, \ldots, d$,

which is not minimal, since it has $d+1$ operators and the rank of the channel is $d$, but it is more convenient for the calculation.

We start by recalling the HKS $\beta_{x}=0$ conditions

$$
i \sum_{l=0}^{d}\left(\partial_{x} K_{l}^{\dagger}\right) K_{l}=-|x\rangle\langle x|=\sum_{i, j=1}^{d} h_{i j}^{x} K_{i}^{\dagger} K_{j} ;
$$

the rhs becomes

$$
\begin{aligned}
& h_{00}^{x} \eta \mathbb{1}+\sum_{j=1}\left(h_{0 j}^{x}+h_{j 0}^{x}\right) \sqrt{\eta(1-\eta)}|j\rangle\langle j| \\
& \quad+\sum_{k, j=1} h_{j k}^{x}(1-\eta) \delta_{j k}|k\rangle\langle k|
\end{aligned}
$$

and we assume that the matrices $h^{x}$ have real elements, obtaining

$$
h_{00}^{x} \eta \mathbb{1}+\sqrt{\eta(1-\eta)} \sum_{j=1} 2 h_{0 j}^{x}|j\rangle\left\langle j\left|+(1-\eta) \sum_{k=1} h_{k k}^{x}\right| k\right\rangle\langle k| .
$$

Now, we make the following Ansatz on the form of the matrices $h^{x}$ : 


$$
\begin{aligned}
& h_{00}^{x}=A, \\
& h_{0 x}^{x}=h_{x 0}^{x}=C, \\
& h_{0 j}^{x}=h_{0 j}^{x}=B, \quad j>0 \wedge j \neq x, \\
& h_{x x}^{x}=F, \\
& h_{x j}^{x}=h_{j x}^{x}=G, \quad j>0 \wedge j \neq x, \\
& h_{j j}^{x}=D, \quad i>0 \wedge j \neq x, \\
& h_{i j}^{x}=h_{j i}^{x}=E, \quad i, j>0 \wedge i, j \neq x,
\end{aligned}
$$

so that all $d$ matrices $\left\{h^{x}\right\}_{x=1}^{d}$ are parametrized by seven real parameters. More explicitly, they look like this

$$
h^{x}=\left[\begin{array}{c|cccccc}
A & B & B & \ldots & C & \ldots & B \\
\hline B & D & E & & G & & E \\
B & E & D & & G & \ldots & E \\
\vdots & & & \ddots & & & \\
C & G & G & & F & & G \\
\vdots & & & \vdots & & \ddots & \\
B & E & E & & G & & D
\end{array}\right],
$$

where the column and row that stand out are the $x$ th ones (starting to count from 0 ). We have numerical evidence that matrices in this form attain the minimum. With this simplification, Eq. (H21) becomes

$$
\begin{aligned}
& A \eta \mathbb{1}+2 B \sqrt{\eta(1-\eta)}(\mathbb{1}-|x\rangle\langle x|)+2 C \sqrt{\eta(1-\eta)}|x\rangle\langle x| \\
& \quad+D(1-\eta)(\mathbb{1}-|x\rangle\langle x|)+F(1-\eta)|x\rangle\langle x|, \quad
\end{aligned}
$$

and the condition $\beta_{x}=0 \forall x$ is satisfied if and only if

$$
\begin{aligned}
& A \eta+2 B \sqrt{\eta(1-\eta)}+D(1-\eta)=0, \\
& A \eta+2 C \sqrt{\eta(1-\eta)}+F(1-\eta)=-1,
\end{aligned}
$$

from which we can eliminate two variables, i.e.,

$$
\begin{gathered}
F=-\frac{1+A \eta+2 C \sqrt{(1-\eta) \eta}}{1-\eta}, \\
D=-\frac{A \eta+2 B \sqrt{(1-\eta) \eta}}{1-\eta} .
\end{gathered}
$$

We obtain

$$
H=\sum_{x=1}^{d}\left(h^{x}\right)^{2}=\left[\begin{array}{c|cccc}
X & Z & Z & \ldots & Z \\
\hline Z & Y & T & & T \\
Z & T & Y & & T \\
\vdots & & & \ddots & \\
Z & T & T & & Y
\end{array}\right],
$$

where

$$
\begin{gathered}
X=d\left[A^{2}+(d-1) B^{2}+C^{2}\right], \\
Y=(d-1)\left[D^{2}+B^{2}+(d-2) E^{2}+G^{2}\right] \\
+C^{2}+F^{2}+(d-1) G^{2}, \\
Z=(d-1)[B(A+D)+(d-2) B E+G C] \\
+C(A+F)+B G(d-1) .
\end{gathered}
$$

Finally, the bound (27) amounts to the following minimization:

$$
\min _{A, B, C, E, G}[X \eta+2 Z \sqrt{(1-\eta) \eta}+(1-\eta) Y-1],
$$

from which we obtain

$$
\mathfrak{B}=\frac{4(d-1) \eta^{2}}{(2+d \eta)(1-\eta)}=\frac{4 \eta}{1-\eta} \frac{d-1}{d+\frac{2}{\eta}}
$$

[1] B. M. Escher, R. L. De Matos Filho, and L. Davidovich, General Framework for Estimating the Ultimate Precision Limit in Noisy Quantum-Enhanced Metrology, Nat. Phys. 7, 406 (2011).

[2] R. Demkowicz-Dobrzański, J. Kołodyński, and M. Guţă, The Elusive Heisenberg Limit in Quantum-Enhanced Metrology, Nat. Commun. 3, 1063 (2012).

[3] R. Demkowicz-Dobrzański and L. Maccone, Using Entanglement against Noise in Quantum Metrology, Phys. Rev. Lett. 113, 250801 (2014).

[4] R. Demkowicz-Dobrzański, J. Czajkowski, and P. Sekatski, Adaptive Quantum Metrology under General Markovian Noise, Phys. Rev. X 7, 041009 (2017).

[5] S. Zhou, M. Zhang, J. Preskill, and L. Jiang, Achieving the Heisenberg Limit in Quantum Metrology Using Quantum Error Correction, Nat. Commun. 9, 78 (2018).

[6] R. Demkowicz-Dobrzański, K. Banaszek, and R. Schnabel, Fundamental Quantum Interferometry Bound for the Squeezed-Light-Enhanced Gravitational Wave Detector GEO 600, Phys. Rev. A 88, 041802(R) (2013).

[7] S. Zhou and L. Jiang, Optimal Approximate Quantum Error Correction for Quantum Metrology, Phys. Rev. Research 2, 013235 (2020).

[8] S. Zhou and L. Jiang, Asymptotic Theory of Quantum Channel Estimation, PRX Quantum 2, 010343 (2021).

[9] M. Tse, H. Yu, N. Kijbunchoo, A. Fernandez-Galiana, P. Dupej, L. Barsotti, C. D. Blair, D. D. Brown, S. E. Dwyer, A. Effler et al., Quantum-Enhanced Advanced LIGO Detectors in the Era of Gravitational-Wave Astronomy, Phys. Rev. Lett. 123, 231107 (2019).

[10] F. Acernese et al. (Virgo Collaboration), Increasing the Astrophysical Reach of the Advanced Virgo Detector via the Application of Squeezed Vacuum States of Light, Phys. Rev. Lett. 123, 231108 (2019). 
[11] T. Baumgratz and A. Datta, Quantum Enhanced Estimation of a Multidimensional Field, Phys. Rev. Lett. 116, 030801 (2016).

[12] P. C. Humphreys, M. Barbieri, A. Datta, and I. A. Walmsley, Quantum Enhanced Multiple Phase Estimation, Phys. Rev. Lett. 111, 070403 (2013).

[13] M. Tsang, H. M. Wiseman, and C. M. Caves, Fundamental Quantum Limit to Waveform Estimation, Phys. Rev. Lett. 106, 090401 (2011).

[14] M. Szczykulska, T. Baumgratz, and A. Datta, MultiParameter Quantum Metrology, Adv. Phys. X 1, 621 (2016).

[15] F. Albarelli, M. Barbieri, M. G. Genoni, and I. Gianani, A Perspective on Multiparameter Quantum Metrology: From Theoretical Tools to Applications in Quantum IImaging, Phys. Lett. A 384, 126311 (2020).

[16] B. M. Terhal, Quantum Error Correction for Quantum Memories, Rev. Mod. Phys. 87, 307 (2015).

[17] S. Pirandola and C. Lupo, Ultimate Precision of Adaptive Noise Estimation, Phys. Rev. Lett. 118, 100502 (2017).

[18] Q. Zhuang and S. Pirandola, Ultimate Limits for Multiple Quantum Channel Discrimination, Phys. Rev. Lett. 125, 080505 (2020).

[19] H. Yuan, Sequential Feedback Scheme Outperforms the Parallel Scheme for Hamiltonian Parameter Estimation, Phys. Rev. Lett. 117, 160801 (2016).

[20] M. Gessner, L. Pezzè, and A. Smerzi, Sensitivity Bounds for Multiparameter Quantum Metrology, Phys. Rev. Lett. 121, 130503 (2018).

[21] N. Kura and M. Ueda, Finite-Error Metrological Bounds on Multiparameter Hamiltonian Estimation, Phys. Rev. A 97, 012101 (2018).

[22] X. Li, J.-H. Cao, Q. Liu, M. K. Tey, and L. You, MultiParameter Estimation with Multi-Mode Ramsey Interferometry, New J. Phys. 22, 043005 (2020).

[23] M. Gessner, A. Smerzi, and L. Pezzè, Multiparameter Squeezing for Optimal Quantum Enhancements in Sensor Networks, Nat. Commun. 11, 3817 (2020).

[24] E. Polino, M. Riva, M. Valeri, R. Silvestri, G. Corrielli, A. Crespi, N. Spagnolo, R. Osellame, and F. Sciarrino, Experimental Multiphase Estimation on a Chip, Optica 6, 288 (2019).

[25] Z. Hou, Z. Zhang, G.-Y. Xiang, C.-F. Li, G.-C. Guo, H. Chen, L. Liu, and H. Yuan, Minimal Tradeoff and Ultimate Precision Limit of Multiparameter Quantum Magnetometry under the Parallel Scheme, Phys. Rev. Lett. 125, 020501 (2020).

[26] Z. Hou, J.-F. Tang, H. Chen, H. Yuan, G.-Y. Xiang, C.-F. Li, and G.-C. Guo, Zero-Trade-Off Multiparameter Quantum Estimation via Simultaneously Saturating Multiple Heisenberg Uncertainty Relations, Sci. Adv. 7, eabd2986 (2021).

[27] Z. Hou, Y. Jin, H. Chen, J.-F. Tang, C.-J. Huang, H. Yuan, G.-Y. Xiang, C.-F. Li, and G.-C. Guo, Super-Heisenberg and Heisenberg Scalings Achieved Simultaneously in the Estimation of a Rotating Field, Phys. Rev. Lett. 126, 070503 (2021).

[28] W. Górecki, S. Zhou, L. Jiang, and R. DemkowiczDobrzański, Optimal Probes and Error-Correction
Schemes in Multi-Parameter Quantum Metrology, Quantum 4, 288 (2020).

[29] S. Ragy, M. Jarzyna, and R. Demkowicz-Dobrzański, Compatibility in Multiparameter Quantum Metrology, Phys. Rev. A 94, 052108 (2016).

[30] C. W. Helstrom, Quantum Detection and Estimation Theory (Academic Press, New York, 1976).

[31] A. S. Holevo, Probabilistic and Statistical Aspects of Quantum Theory, 2nd ed. (Edizioni della Normale, Pisa, 2011).

[32] M. G. A. Paris, Quantum Estimation for Quantum Technology, Int. J. Quantum. Inform. 07, 125 (2009).

[33] J. Liu, H. Yuan, X.-M. Lu, and X. Wang, Quantum Fisher Information Matrix and Multiparameter Estimation, J. Phys. A 53, 023001 (2020).

[34] R. Demkowicz-Dobrzański, W. Górecki, and M. Guţă, Multi-Parameter Estimation beyond Quantum Fisher Information, J. Phys. A 53, 363001 (2020).

[35] H. P. Yuen and M. Lax, Multiple-Parameter Quantum Estimation and Measurement of Nonselfadjoint Observables, IEEE Trans. Inf. Theory 19, 740 (1973).

[36] V. P. Belavkin and B. A. Grishanin, Optimum Estimation in Quantum Channels by the Generalized Heisenberg Inequality Method, Prob. Peredachi Inf. 9, 44 (1973).

[37] A. S. Holevo, in Proceedings of the Third Japan-USSR Symposium on Probability Theory, edited by G. Maruyama and J. V. Prokhorov (Springer, Berlin, 1976), Vol. 550.

[38] K. Matsumoto, A New Approach to the Cramér-Rao-Type Bound of the Pure-State Model, J. Phys. A 35, 3111 (2002).

[39] L. Pezzè, M. A. Ciampini, N. Spagnolo, P. C. Humphreys, A. Datta, I. A. Walmsley, M. Barbieri, F. Sciarrino, and A. Smerzi, Optimal Measurements for Simultaneous Quantum Estimation of Multiple Phases, Phys. Rev. Lett. 119, 130504 (2017).

[40] F. Albarelli, J. F. Friel, and A. Datta, Evaluating the Holevo Cramér-Rao Bound for Multiparameter Quantum Metrology, Phys. Rev. Lett. 123, 200503 (2019).

[41] J. Yang, S. Pang, Y. Zhou, and A. N. Jordan, Optimal Measurements for Quantum Multiparameter Estimation with General States, Phys. Rev. A 100, 032104 (2019).

[42] J. S. Sidhu, Y. Ouyang, E. T. Campbell, and P. Kok, Tight Bounds on the Simultaneous Estimation of Incompatible Parameters, Phys. Rev. X 11, 011028 (2021).

[43] S. Razavian, M. G. A. Paris, and M. G. Genoni, On the Quantumness of Multiparameter Estimation Problems for Qubit Systems, Entropy 22, 1197 (2020).

[44] X.-M. Lu and X. Wang, Incorporating Heisenberg's Uncertainty Principle into Quantum Multiparameter Estimation, Phys. Rev. Lett. 126, 120503 (2021).

[45] L. O. Conlon, J. Suzuki, P. K. Lam, and S. M. Assad, Efficient Computation of the Nagaoka-Hayashi Bound for Multiparameter Estimation with Separable Measurements, npj Quantum Inf. 7, 110 (2021).

[46] F. Belliardo and V. Giovannetti, Incompatibility in Quantum Parameter Estimation, New J. Phys. 23, 063055 (2021).

[47] A. Carollo, B. Spagnolo, A. A. Dubkov, and D. Valenti, On Quantumness in Multi-Parameter Quantum Estimation, J. Stat. Mech. (2019) 094010. 
[48] M. Tsang, F. Albarelli, and A. Datta, Quantum Semiparametric Estimation, Phys. Rev. X 10, 031023 (2020).

[49] J. Kahn and M. Guţă, Local Asymptotic Normality for Finite Dimensional Quantum Systems, Commun. Math. Phys. 289, 597 (2009).

[50] K. Yamagata, A. Fujiwara, and R. D. Gill, Quantum Local Asymptotic Normality Based on a New Quantum Likelihood Ratio, Ann. Stat. 41, 2197 (2013).

[51] Y. Yang, G. Chiribella, and M. Hayashi, Attaining the Ultimate Precision Limit in Quantum State Estimation, Commun. Math. Phys. 368, 223 (2019).

[52] M. Jarzyna and R. Demkowicz-Dobrzański, Matrix Product States for Quantum Metrology, Phys. Rev. Lett. 110, 240405 (2013).

[53] K. Chabuda, J. Dziarmaga, T. J. Osborne, and R. Demkowicz-Dobrzanski, Tensor-Network Approach for Quantum Metrology in Many-Body Quantum Systems, Nat. Commun. 11, 250 (2020).

[54] M. Tsang, Quantum Metrology with Open Dynamical Systems, New J. Phys. 15, 073005 (2013).

[55] J.-D. Yue, Y.-r. Zhang, and H. Fan, Quantum-Enhanced Metrology for Multiple Phase Estimation with Noise, Sci. Rep. 4, 5933 (2015).

[56] M. D. Vidrighin, G. Donati, M. G. Genoni, X.-M. Jin, W. S. Kolthammer, M. S. Kim, A. Datta, M. Barbieri, and I. A. Walmsley, Joint Estimation of Phase and Phase Diffusion for Quantum Metrology, Nat. Commun. 5, 3532 (2014).

[57] P. J. D. Crowley, A. Datta, M. Barbieri, and I. A. Walmsley, Tradeoff in Simultaneous Quantum-Limited Phase and Loss Estimation in Interferometry, Phys. Rev. A 89, 023845 (2014).

[58] L. B. Ho, H. Hakoshima, Y. Matsuzaki, M. Matsuzaki, and Y. Kondo, Multiparameter Quantum Estimation under Dephasing Noise, Phys. Rev. A 102, 022602 (2020).

[59] J. Friel, P. Palittapongarnpim, F. Albarelli, and A. Datta, Attainability of the Holevo-Cramér-Rao Bound for TwoQubit 3D Magnetometry, arXiv:2008.01502.

[60] A. Fujiwara and H. Imai, A Fibre Bundle over Manifolds of Quantum Channels and Its Application to Quantum Statistics, J. Phys. A 41, 255304 (2008).

[61] R. Demkowicz-Dobrzański and M. Markiewicz, Quantum Computation Speedup Limits from Quantum Metrological Precision Bounds, Phys. Rev. A 91, 062322 (2015).

[62] V. Katariya and M. M. Wilde, RLD Fisher Information Bound for Multiparameter Estimation of Quantum Channels, New J. Phys. 23, 073040 (2021).

[63] Y. Chen and H. Yuan, Maximal Quantum Fisher Information Matrix, New J. Phys. 19, 063023 (2017).

[64] Asymptotic Theory of Quantum Statistical Inference, edited by M. Hayahsi (World Scientific, Singapore, 2005).

[65] S. D. Silvey, Optimal Design: An Introduction to the Theory for Parameter Estimation (Springer Netherlands, Dordrecht, 1980).

[66] M. A. Ballester, Entanglement Is Not Very Useful for Estimating Multiple Phases, Phys. Rev. A 70, 032310 (2004).

[67] W. Ge, K. Jacobs, Z. Eldredge, A. V. Gorshkov, and M. Foss-Feig, Distributed Quantum Metrology with Linear Networks and Separable Inputs, Phys. Rev. Lett. 121, 043604 (2018).
[68] A. Ambainis, D. Leung, L. Mancinska, and M. Ozols, Quantum Random Access Codes with Shared Randomness, arXiv:0810.2937.

[69] A. Tavakoli, A. Hameedi, B. Marques, and M. Bourennane, Quantum Random Access Codes Using Single d-Level Systems, Phys. Rev. Lett. 114, 170502 (2015).

[70] B. Yadin, M. Fadel, and M. Gessner, Metrological Complementarity Reveals the Einstein-Podolsky-Rosen Paradox, Nat. Commun. 12, 2410 (2021).

[71] J. Suzuki, Y. Yang, and M. Hayashi, Quantum State Estimation with Nuisance Parameters, J. Phys. A 53, 453001 (2020).

[72] J. Kołodyński and R. Demkowicz-Dobrzański, Efficient Tools for Quantum Metrology with Uncorrelated Noise, New J. Phys. 15, 073043 (2013).

[73] github.com/falbarelli/multipar-purification-bounds.

[74] R. T. Rockafellar, Convex Analysis (Princeton University Press, Princeton, NJ, 1970).

[75] P. Sekatski, M. Skotiniotis, J. Kołodyński, and W. Dür, Quantum Metrology with Full and Fast Quantum Control, Quantum 1, 27 (2017).

[76] To see explicitly that this is possible, we can choose without loss of generality $u_{\boldsymbol{\theta}}=e^{-i \sum_{j=1}^{p}\left(\theta_{j}-\theta_{j}^{*}\right) h_{j}}$, where $\boldsymbol{\theta}^{*}$ is the true value of the parameters at which all derivatives and functions are evaluated and indeed, $\left.u_{\theta}^{\dagger} \partial_{j} u_{\theta}\right|_{\theta=\theta^{*}}=-i h_{j}$.

[77] J. Kołodyński, Precision Bounds in Noisy Quantum Metrology, Ph.D. thesis, University of Warsaw, 2014, arXiv:1409.0535.

[78] D. Petz, Monotone Metrics on Matrix Spaces, Linear Algebra Appl. 244, 81 (1996).

[79] J. Suzuki, Information Geometrical Characterization of Quantum Statistical Models in Quantum Estimation Theory, Entropy 21, 703 (2019).

[80] M. A. Ballester, Estimation of Unitary Quantum Operations, Phys. Rev. A 69, 022303 (2004).

[81] J. Kahn, Fast Rate Estimation of a Unitary Operation in SU(d), Phys. Rev. A 75, 022326 (2007).

[82] H. Imai and A. Fujiwara, Geometry of Optimal Estimation Scheme for SU(D) Channels, J. Phys. A 40, 4391 (2007).

[83] C. Macchiavello, Optimal Estimation of Multiple Phases, Phys. Rev. A 67, 062302 (2003).

[84] C. N. Gagatsos, D. Branford, and A. Datta, Gaussian Systems for Quantum-Enhanced Multiple Phase Estimation, Phys. Rev. A 94, 042342 (2016).

[85] L. Zhang and K. W. C. Chan, Quantum Multiparameter Estimation with Generalized Balanced Multimode NOONlike States, Phys. Rev. A 95, 032321 (2017).

[86] C. You, S. Adhikari, Y. Chi, M. L. LaBorde, C. T. Matyas, C. Zhang, Z. Su, T. Byrnes, C. Lu, J. P. Dowling, and J. P. Olson, Multiparameter Estimation with Single PhotonsLinearly-Optically Generated Quantum Entanglement Beats the Shotnoise Limit, J. Opt. 19, 124002 (2017).

[87] A.Z. Goldberg, I. Gianani, M. Barbieri, F. Sciarrino, A. M. Steinberg, and N. Spagnolo, Multiphase Estimation without a Reference Mode, Phys. Rev. A 102, 022230 (2020).

[88] M. Markiewicz, M. Pandit, and W. Laskowski, Simultaneous Estimation of Multiple Phases in Generalised Mach-Zehnder Interferometer, Sci. Rep. 11, 15669 (2021). 
[89] M. Valeri, E. Polino, D. Poderini, I. Gianani, G. Corrielli, A. Crespi, R. Osellame, N. Spagnolo, and F. Sciarrino, Experimental Adaptive Bayesian Estimation of Multiple Phases with Limited Data, npj Quantum Inf. 6, 92 (2020).

[90] W. Gorecki and R. Demkowicz-Dobrzanski, MultiplePhase Quantum Interferometry - Real and Apparent Gains of Measuring All the Phases Simultaneously, arXiv: 210710863.

[91] R. Demkowicz-Dobrzański, M. Jarzyna, and J. Kołodyński, Quantum Limits in Optical Interferometry, Prog. Opt. 60, 345 (2015).

[92] It is enough to compute the QFI of a single-mode Gaussian state, which gives a bound generally saturable by phasesensitive measurements, e.g., homodyne detection, where a reference phase is taken for granted [93].

[93] M. Jarzyna and R. Demkowicz-Dobrzański, Quantum Interferometry with and without an External Phase Reference, Phys. Rev. A 85, 011801(R) (2012).

[94] P. M. Birchall, E. J. Allen, T. M. Stace, J. L. O’Brien, J. C. F. Matthews, and H. Cable, Quantum Optical Metrology of Correlated Phase and Loss, Phys. Rev. Lett. 124, 140501 (2020).

[95] R. Nair, Quantum-Limited Loss Sensing: Multiparameter Estimation and Bures Distance between Loss Channels, Phys. Rev. Lett. 121, 230801 (2018).

[96] G. Adesso, F. Dell'Anno, S. De Siena, F. Illuminati, and L. A. M. Souza, Optimal Estimation of Losses at the Ultimate Quantum Limit with Non-Gaussian States, Phys. Rev. A 79, 040305(R) (2009).

[97] S. I. Knysh and G. A. Durkin, Estimation of Phase and Diffusion: Combining Quantum Statistics and Classical Noise, arXiv:1307.0470.

[98] M. Szczykulska, T. Baumgratz, and A. Datta, Reaching for the Quantum Limits in the Simultaneous Estimation of Phase and Phase Diffusion, Quantum Sci. Technol. 2, 044004 (2017).

[99] J. Watrous, The Theory of Quantum Information (Cambridge University Press, Cambridge, England, 2018).

[100] C. Fuchs and J. van de Graaf, Cryptographic Distinguishability Measures for Quantum-Mechanical States, IEEE Trans. Inf. Theory 45, 1216 (1999).

[101] D. Qiu, Minimum-Error Discrimination between Mixed Quantum States, Phys. Rev. A 77, 012328 (2008).

[102] I. Bengtsson and K. Życzkowski, Geometry of Quantum States: An Introduction to Quantum Entanglement, 2nd ed. (Cambridge University Press, Cambridge, England, 2017).

[103] A. Uhlmann, in Groups and Related Topics, edited by R. Gielerak, J. Lukierski, and Z. Popowicz (Springer Netherlands, Dordrecht, 1992), pp. 267-274.

[104] M. M. Taddei, B. M. Escher, L. Davidovich, and R. L. de Matos Filho, Quantum Speed Limit for Physical Processes, Phys. Rev. Lett. 110, 050402 (2013).

[105] A. W. Harrow and A. Winter, How Many Copies Are Needed for State Discrimination?, IEEE Trans. Inf. Theory 58, 1 (2012).

[106] R. Duan, Y. Feng, and M. Ying, Perfect Distinguishability of Quantum Operations, Phys. Rev. Lett. 103, 210501 (2009).

[107] Y. Chen and H. Yuan, Zero-Error Quantum Hypothesis Testing in Finite Time with Quantum Error Correction, Phys. Rev. A 100, 022336 (2019).
[108] A. Acín, Statistical Distinguishability between Unitary Operations, Phys. Rev. Lett. 87, 177901 (2001).

[109] E. Farhi and S. Gutmann, Analog Analogue of a Digital Quantum Computation, Phys. Rev. A 57, 2403 (1998).

[110] O. Regev and L. Schiff, in Automata, Languages and Programming (Springer, Berlin, 2008), pp. 773-781.

[111] K. Temme, Runtime of Unstructured Search with a Faulty Hamiltonian Oracle, Phys. Rev. A 90, 022310 (2014).

[112] T. Heinosaari, T. Miyadera, and M. Ziman, An Invitation to Quantum Incompatibility, J. Phys. A 49, 123001 (2016).

[113] T. Heinosaari and T. Miyadera, Incompatibility of Quantum Channels, J. Phys. A 50, 135302 (2017).

[114] M. Girard, M. Plávala, and J. Sikora, Jordan Products of Quantum Channels and Their Compatibility, Nat. Commun. 12, 2129 (2021).

[115] A. Altherr and Y. Yang, Quantum Metrology for NonMarkovian Processes, Phys. Rev. Lett. 127, 060501 (2021).

[116] M. J. W. Hall and H. M. Wiseman, Does Nonlinear Metrology Offer Improved Resolution? Answers from Quantum Information Theory, Phys. Rev. X 2, 041006 (2012).

[117] M. Jarzyna and R. Demkowicz-Dobrzański, True Precision Limits in Quantum Metrology, New J. Phys. 17, 013010 (2015).

[118] W. Górecki, R. Demkowicz-Dobrzański, H. M. Wiseman, and D. W. Berry, $\pi$-Corrected Heisenberg Limit, Phys. Rev. Lett. 124, 030501 (2020).

[119] G. Guarnieri, G. T. Landi, S. R. Clark, and J. Goold, Thermodynamics of Precision in Quantum Nonequilibrium Steady States, Phys. Rev. Research 1, 033021 (2019).

[120] Y. Hasegawa, Quantum Thermodynamic Uncertainty Relation for Continuous Measurement, Phys. Rev. Lett. 125, 050601 (2020).

[121] S. Deffner and S. Campbell, Quantum Speed Limits: From Heisenberg's Uncertainty Principle to Optimal Quantum Control, J. Phys. A 50, 453001 (2017).

[122] J. J. Meyer, Fisher Information in Noisy IntermediateScale Quantum Applications, Quantum 5, 539 (2021).

[123] A. Kubica and R. Demkowicz-Dobrzański, Using Quantum Metrological Bounds in Quantum Error Correction: A Simple Proof of the Approximate Eastin-Knill Theorem, Phys. Rev. Lett. 126, 150503 (2021).

[124] S. Zhou, Z.-W. Liu, and L. Jiang, New Perspectives on Covariant Quantum Error Correction, Quantum 5, 521 (2021).

[125] Y. Yang, Y. Mo, J. M. Renes, G. Chiribella, and M. P. Woods, Covariant Quantum Error Correcting Codes via Reference Frames, arXiv:2007.09154.

[126] M. Hayashi, Comparison between the Cramer-Rao and the Mini-Max Approaches in Quantum Channel Estimation, Commun. Math. Phys. 304, 689 (2011).

[127] V. Katariya and M. M. Wilde, Geometric Distinguishability Measures Limit Quantum Channel Estimation and Discrimination, Quantum Inf. Process. 20, 78 (2021).

[128] M. A. Nielsen and I. L. Chuang, Quantum Computation and Quantum Information, 10th ed. (Cambridge University Press, Cambridge, England, 2010).

[129] A. Fujiwara, Estimation of a Generalized AmplitudeDamping Channel, Phys. Rev. A 70, 012317 (2004). 